\title{
Graded Bundles in the Category of Lie Groupoids ${ }^{\star}$
}

\author{
Andrew James BRUCE ${ }^{\dagger}$, Katarzyna GRABOWSKA ${ }^{\ddagger}$ and Janusz GRABOWSKI ${ }^{\dagger}$ \\ $\dagger$ Institute of Mathematics, Polish Academy of Sciences, Poland \\ E-mail: andrewjamesbruce@googlemail.com,jagrab@impan.pl \\ $\ddagger$ Faculty of Physics, University of Warsaw, Poland \\ E-mail: konieczn@fuw.edu.pl
}

Received February 25, 2015, in final form November 05, 2015; Published online November 11, 2015 http://dx.doi.org/10.3842/SIGMA.2015.090

\begin{abstract}
We define and make initial study of Lie groupoids equipped with a compatible homogeneity (or graded bundle) structure, such objects we will refer to as weighted Lie groupoids. One can think of weighted Lie groupoids as graded manifolds in the category of Lie groupoids. This is a very rich geometrical theory with numerous natural examples. Note that $\mathcal{V B}$-groupoids, extensively studied in the recent literature, form just the particular case of weighted Lie groupoids of degree one. We examine the Lie theory related to weighted groupoids and weighted Lie algebroids, objects defined in a previous publication of the authors, which are graded manifolds in the category of Lie algebroids, showing that they are naturally related via differentiation and integration. In this work we also make an initial study of weighted Poisson-Lie groupoids and weighted Lie bi-algebroids, as well as weighted Courant algebroids.
\end{abstract}

Key words: graded manifolds; homogeneity structures; Lie groupoids; Lie algebroids

2010 Mathematics Subject Classification: 22A22; 55R10; 58E40; 58H05

\section{Introduction}

Lie groupoids and Lie algebroids are ubiquitous throughout differential geometry and are playing an ever increasing rôle in mathematical physics. Lie groupoids provide a unifying framework to discuss diverse topics in modern geometry including the theory of group actions, foliations, Poisson geometry, orbifolds, principal bundles, connection theory and so on. The infinitesimal counterpart to Lie groupoids are Lie algebroids. The Lie theory here is very rich and not as simple as the corresponding Lie theory for Lie groups and Lie algebras. In particular, while it is true that every Lie groupoid can be differentiated to obtain a Lie algebroid, the reverse procedure of global integration has an obstruction [7,26]. Not all Lie algebroids can be globally integrated to obtain a Lie groupoid, although one can always integrate Lie algebroids to 'local' Lie groupoids.

Another important notion in modern geometry is that of a graded manifold. Such notions have their conception in the 'super' context via the BV-BRST formalism of gauge theories in physics. We remark that Lie algebroids appear in physics as symmetries of field theories that do not arise from Lie groups or Lie algebras. Moreover, these symmetries cannot always be directly separated from the space of fields. Such symmetries are naturally accommodated in the BV-BRST formalism. The concept of a graded manifold has been put to good use in describing Lie algebroids, Lie bi-algebroids, Courant algebroids and related notions [38, 39, 44, 46]. In the purely commutative setting, Grabowski and Rotkiewicz [15] define what they referred to as

\footnotetext{
*This paper is a contribution to the Special Issue on Poisson Geometry in Mathematics and Physics. The full collection is available at http://www.emis.de/journals/SIGMA/Poisson2014.html
} 
graded bundles. Loosely, a graded bundle is a natural generalisation of the concept of a vector bundle. We will discuss graded bundles in a little more detail shortly.

There has been some recent interest in $\mathcal{V B}$-groupoids and $\mathcal{V} \mathcal{B}$-algebroids, see for example $[1,5,16,17]$. From a categorical point of view $\mathcal{V} \mathcal{B}$-groupoids are vector bundles in the category of Lie groupoids and similarly $\mathcal{V B}$-algebroids are vector bundles in the category of Lie algebroids. It is known, via $[1,5]$, that the Lie functor restricts to the category of $\mathcal{V B}$-groupoids and $\mathcal{V} \mathcal{B}$ algebroids; that is we can differentiate $\mathcal{V B}$-groupoids to get $\mathcal{V} \mathcal{B}$-algebroids and $\mathcal{V B}$-algebroids integrate to $\mathcal{V B}$-groupoids. Let us mention that $\mathcal{V B}$-groupoids and $\mathcal{V} \mathcal{B}$-algebroids have been studied in relation to Poisson geometry and representations. Indeed, representations of Lie groupoids on vector bundles gives rise to $\mathcal{V B}$-groupoids.

As graded bundles are generalisations of vector bundles, it seems natural that one should study graded bundles in the category of Lie groupoids and Lie algebroids, including their Lie theory. The first work in this direction was by the authors of this paper, where various descriptions of so-called weighted Lie algebroids were given in [3]. The motivation for that work was to uncover a practical notion of a "higher Lie algebroid" suitable for higher-order geometric mechanics in the spirit of Tulczyjew. We presented details of the Lagrangian and Hamiltonian formalisms on graded bundles using weighted Lie algebroids in a separate publication [2]. As an application, the higher-order Euler-Lagrange equations on a Lie algebroid were derived completely geometrically, and in full agreement with the independent approach of Martínez using variational calculus [32]. This and other results have convinced us of the potential for further applications of weighted Lie algebroids.

The natural question of integrating weighted Lie algebroids was not posed at all in [3] or [2]. We address this question in this paper. We define weighted Lie groupoids as Lie groupoids that carry a compatible homogeneity structure, i.e., an action of the monoid $\mathbb{R}$ of multiplicative reals; we will make this precise in due course. We show that these structures, which are a generalisation of $\mathcal{V B}$-groupoids, are the objects that integrate weighted Lie algebroids. Just as the tangent bundle of a Lie groupoid is a canonical example of a $\mathcal{V B}$-groupoid, the $k$-th order higher tangent bundle of a Lie groupoid is a canonical example of a weighted Lie groupoid.

The results found in this paper can be viewed as the 'higher order' generalisations of the results found in [5]. In particular, Bursztyn et al. [5] treat $\mathcal{V} \mathcal{B}$-groupoids/algebroids as Lie groupoids/algebroids equipped with a compatible regular homogeneity structure. The idea of simplifying $\mathcal{V B}$-'objects' by describing then in terms of regular homogeneity structures goes back to [14], where symplectic structures were studied in this context. By passing to graded bundles, we do not really further simplify the notion of $\mathcal{V B}$-'objects', but by understanding them as part of a larger class of structures we simplify working with them.

Following our intuition, we also examine the notion of weighted Poisson-Lie groupoids as Poisson-Lie groupoids equipped with a compatible homogeneity structure. The infinitesimal versions of weighted Poisson-Lie groupoids are weighted Lie bi-algebroids, a notion we carefully define in this paper. Associated with any Lie bi-algebroid is a Courant algebroid. We show that this notion very naturally passes over to the weighted case and motivates a more general notion of a weighted Courant algebroid, which is a natural generalisation of a $\mathcal{V B}$-Courant algebroid. The use of a compatible homogeneity structure provokes the question of replacing the monoid $\mathbb{R}$ by its subgroup $\mathbb{R}^{\times}$of multiplicative reals $\neq 0$. We claim that this is a natural way of defining contact and Jacobi groupoids, see [4].

Summing up, one can say that in this paper we generalise the notion of $\mathcal{V B}$-'objects' by passing to the category of graded bundles and categorical objects therein. On the other hand, these concepts and their applications are far from being fully exploited. This work is only the first step in this direction.

Our use of supermanifolds. We recognise the power and elegance of the framework of supermanifolds in the context of algebroid-like objects and we will exploit this formalism. In 
particular we will make use of the so called $Q$-manifolds, that is supermanifolds equipped with a homological vector field. Although we will not dwell on fundamental issues from the theory of supermanifolds, we will technically follow the "Russian school" and understand supermanifolds in terms of locally superringed spaces. However, for the most part the intuitive and correct understanding of a supermanifold as a 'manifold' with both commuting and anticommuting coordinates will suffice. When necessary we will denote the Grassmann parity of an object by 'tilde'.

Arrangement of paper. In Section 2 we briefly recall the necessary parts of theory of graded bundles, weighted Lie algebroids and Lie groupoids as needed in the rest of this paper. In Section 3 we introduce the main objects of study, that is weighted Lie groupoids. Weighted Lie theory is the subject of Section 4. Finally in Section 5 we apply some of the ideas developed earlier in this paper to weighted Poisson-Lie groupoids, weighted Lie bi-algebroids and weighted Courant algebroids. We end with some remarks on contact and Jacobi groupoids.

\section{Preliminaries}

In this section we briefly recall parts of the theory of graded bundles, $n$-tuple graded bundles and weighted algebroids as needed in later sections of this paper. Everything in this paper will be in the smooth category. The interested reader should consult the original literature $[3,12,14,15]$ for details, such as proofs of the statements made in this section. We will also set some notation regarding Lie groupoids and recall the groupoid/algebroid version of Lie's second theorem, which was first proved by Mackenzie and $\mathrm{Xu}$ [30]. For an overview of the general theory of Lie groupoids and Lie algebroids the reader can consult Mackenzie [28]. We will also very briefly recall the notion of a $Q$-manifold and a $Q S$-manifold as needed throughout this paper.

\subsection{Graded and $n$-tuple graded bundles}

Manifolds and supermanifolds that carry various extra gradings on their structure sheaf are now an established part of modern geometry and mathematical physics. The general theory of graded manifolds in our understanding was formulated by Voronov in [44]; we must also mention the works of Kontsevich [21], Mehta [33], Roytenberg [38] and Ševera [41] where various notions of a $\mathbb{Z}$-graded supermanifold appear. The graded structure on such (super)manifolds is conveniently encoded in the weight vector field whose action via the Lie derivative counts the degree of tensor and tensor-like objects on the (super)manifold.

In this introductory section we will concentrate our attention on just genuine manifold and only sketch the theory for supermanifolds, as the extension of the results here to supermanifolds is almost straightforward. In later sections we will make use of supermanifolds that carry additional gradings coming from a homogeneity structure which will be defined in a moment.

An important class of graded manifolds are those that carry non-negative grading. It will be convenient to denote homogeneous local coordinates in the form $\left(y_{w}^{a}\right)$ (or $\left(y_{a}^{w}\right)$ ), where $w=$ $0,1, \ldots, k$ is the weight of $y_{a}^{w}$. A canonical example of such a structure is the bundle $\mathrm{T}^{k} M=$ $J_{0}^{k}(\mathbb{R}, M)$ of $k$-velocities, i.e., $k$ th-jets (at 0 ) of curves $\gamma: \mathbb{R} \rightarrow M$. We will furthermore require that (like in the case of $\mathrm{T}^{k} M$ ) this grading is associated with a smooth action $h: \mathbb{R}_{\geq 0} \times F \rightarrow F$ of the monoid $\left(\mathbb{R}_{\geq 0}, \cdot\right)$ of multiplicative non-negative reals on a manifold $F$. Let us recall in this context that a function $f \in C^{\infty}(F)$ we call homogeneous of degree $k \in \mathbb{R}$ if

$$
h_{t}^{*}(f):=f(h(t, \cdot))=t^{k} f
$$

for all $t>0$. We call $k$ the weight or degree of $f$.

Such actions are known as homogeneity structures in the terminology of Grabowski and Rotkiewicz [15] who proved that only non-negative integer weights are allowed, so the algebra $\mathcal{A}(F) \subset C^{\infty}(F)$ spanned by homogeneous function has a canonical $\mathbb{N}$-grading, $\mathcal{A}(F)=$ 
$\bigoplus_{i \in \mathbb{N}} \mathcal{A}^{i}(F)$. This algebra is referred to as the algebra of polynomial functions on $F$. This action reduced to $\mathbb{R}_{>0}$ is the one-parameter group of diffeomorphism integrating the weight vector field, thus the weight vector field is in this case h-complete [12]. Note also that the homogeneity structure always has a canonical extension to the action $h: \mathbb{R} \times F \rightarrow F$ of the monoid $(\mathbb{R}, \cdot)$ such that any homogeneous function $f$ of degree $k \in \mathbb{N}$ satisfies (2.1) this time for all $t \in \mathbb{R}$; it will be convenient to speak about a homogeneity structure as this extended action.

Importantly, we have that for $t \neq 0$ the action $h_{t}$ is a diffeomorphism of $F$ and when $t=0$ it is a smooth surjection $\tau=h_{0}$ onto a submanifold $F_{0}=M$, with the fibres being diffeomorphic to $\mathbb{R}^{N}$ (cf. [15]). Thus, the objects obtained are particular kinds of polynomial bundles $\tau: F \rightarrow M$, i.e., fibrations which locally look like $U \times \mathbb{R}^{N}$ and the change of coordinates (for a certain choice of an atlas) are polynomial in $\mathbb{R}^{N}$. For this reason graded manifolds with non-negative weights and h-complete weight vector fields are also known as graded bundles [15]. Furthermore, the h-completeness condition implies that graded bundles are determined by the algebra of homogeneous functions on them. Canonical examples of graded bundles are higher tangent bundles $\mathrm{T}^{k} M$. The canonical coordinates $\left(x^{a}, \dot{x}^{b}, \ddot{x}^{c}, \ldots\right)$ on $\mathrm{T}^{k} M$, associated with local coordinates $\left(x^{a}\right)$ on $M$, have degrees, respectively, $0,1,2, \ldots$, so the homogeneity structure reads

$$
h_{t}^{*}\left(x^{a}, \dot{x}^{b}, \ddot{x}^{c}, \ldots\right)=\left(x^{a}, t \dot{x}^{b}, t^{2} \ddot{x}^{c}, \ldots\right) .
$$

A fundamental result is that any smooth action of the multiplicative monoid $(\mathbb{R}, \cdot)$ on a manifold leads to a $\mathbb{N}$-gradation of that manifold. A little more carefully, the category of graded bundles is equivalent to the category of $(\mathbb{R}, \cdot)$-manifolds and equivariant maps. A canonical construction of this correspondence goes as follows. Take $p \in F$ and consider $t \mapsto h_{t}(p)$ as a smooth curve $\gamma_{h}^{p}$ in $F$. This curve meets $M$ for $t=0$ and is constant for $p \in M$.

Theorem 2.1 ([15]). For any homogeneity structure $h: \mathbb{R} \times F \rightarrow F$ there is $k \in \mathbb{N}$ such that the map

$$
\Phi_{h}^{k}: \quad F \rightarrow \mathrm{T}^{k} F_{\mid M}, \quad \Phi_{h}^{k}(p)=\mathrm{j}_{0}^{k}\left(\gamma_{h}^{p}\right),
$$

is an equivariant (with respect to the monoid actions of $(\mathbb{R}, \cdot)$ on $F$ and $\mathrm{T}^{k} F$ ) embedding of $F$ into a graded submanifold of the graded bundle $\mathrm{T}^{k} F_{\mid M}$. In particular, there is an atlas on $F$ consisting of homogeneous function.

Any $k$ described by the above theorem we call a degree of the homogeneity structure $h$. We stress that a graded bundle is not just a manifold with consistently defined homogeneous local coordinates. The additional condition is that the weight vector field encoding the graded structure is h-complete; that is the associated one-parameter group of diffeomorphisms can be extended to an action of the monoid $(\mathbb{R}, \cdot)$.

Remark 2.2. The above theorem has a counterpart for graded supermanifolds, the proof of which we will quickly sketch here. Following Voronov [44], we do not require that weights induce parity. The compatibility condition just means that $\mathbb{N}$-weights commute with $\mathbb{Z}_{2}$-grading. In other words, a homogeneity structure on a supermanifold $\mathcal{M}$ is a smooth action $h: \mathbb{R} \times \mathcal{M} \rightarrow \mathcal{M}$ of the monoid $(\mathbb{R}, \cdot)$ such that $h_{t}: \mathcal{M} \rightarrow \mathcal{M}$ are morphisms, i.e., respect the parity. Equivalently, we have an $(\mathbb{R}, \cdot)$-action on the super-algebra $\mathcal{O}_{\mathcal{M}}=C^{\infty}(\mathcal{M})$ by homomorphisms.

The method of the proof of [20, Theorem 1.13] can be easily adapted to the supermanifold case to show that $\mathcal{M}_{0}=h_{0}(\mathcal{M})$ is a submanifold of $\mathcal{M}$. We can also adapt the methods of the proofs of Lemma 4.1 and Theorem 4.1 in [15]. First, define $H(t): \mathrm{T} \mathcal{M}_{\mid \mathcal{M}_{0}} \rightarrow \mathrm{T} \mathcal{M}_{\mid \mathcal{M}_{0}}$ as the derivative $\mathrm{T} h_{t}$ restricted to $\mathrm{T} \mathcal{M}_{\mid \mathcal{M}_{0}}$. We have $H(t) H(s)=H(t s)$, so $Q_{0}=H(0)$ is a projector. Since both, $Q_{0}$ and $\mathbb{1}-Q_{0}$ are projectors whose rank cannot fall locally, the rank of $Q_{0}$ is constant along $\mathcal{M}_{0}$ (assuming $\mathcal{M}$ is connected) and $E_{0}=Q_{0}\left(\mathrm{~T} \mathcal{M}_{\mid \mathcal{M}_{0}}\right)$ is a vector subbundle 
of $\mathrm{T} \mathcal{M}_{\mid \mathcal{M}_{0}}$. One can show inductively that $Q_{r}=\frac{\mathrm{d}^{r} H}{\mathrm{~d} t^{r}}(0)$ is a projector operator defined on the kernel of $Q_{r-1}$ and we put $E_{r}$ to be its range. According to the argument similar to that of [15, Proposition 4.2], $E_{k+1}=\mathrm{T} \mathcal{M}_{0}$ for some $k$. We have therefore a vector bundle decomposition

$$
\mathrm{T} \mathcal{M}_{\mid \mathcal{M}_{0}}=E_{0} \oplus_{\mathcal{M}_{0}} \cdots \oplus_{\mathcal{M}_{0}} E_{k+1}
$$

such that, roughly speaking,

$$
\frac{\partial}{\partial x} \frac{\partial^{i} h}{\partial t^{i}}(0, x)
$$

viewed as a liner map on $\mathrm{T} \mathcal{M}_{\mid \mathcal{M}_{0}}$, vanish on $E_{r}$ for $r>i$ and are identical on $E_{i}$. This implies that the analog of the map $(2.2), \Phi_{h}^{k}: \mathcal{M} \rightarrow \mathrm{T}^{k} \mathcal{M}_{\mid \mathcal{M}_{0}}$. whose coordinate form is

$$
\Phi_{h}^{k}(x)=\left(h(0, x), \frac{\partial h}{\partial t}(0, x), \ldots, \frac{\partial^{k} h}{\partial t^{k}}(0, x)\right),
$$

is of maximal rank at $\mathcal{M}_{0}$. Since it intertwines the actions of $(\mathbb{R}, \cdot)($ cf. [15, Lemma 4.1]), it is actually an embedding into a graded submanifold of $\mathrm{T}^{k} \mathcal{M}_{\mid \mathcal{M}_{0}}$. Indeed, the maximal rank argument is sufficient for odd coordinates, while for even ones we can refer to [15].

Morphisms between graded bundles are necessarily polynomial in the non-zero weight coordinates and respect the weight. Such morphisms can be characterised by the fact that they relate the respective weight vector fields, or equivalently the respective homogeneity structures [15].

Remark 2.3. It is possible to consider manifolds with gradations that do not lead to h-complete weight vector fields. From the point of view of this paper such manifolds are less rigid in their structure and will exhibit pathological behavior. We remark that the definition given by Voronov [44] of a non-negatively graded supermanifold states that the Grassmann even coordinates are 'cylindrical'. Similarly, Roytenberg [38] also insists on this 'cylindrical' condition in his definition of an $\mathrm{N}$-manifold. This together with the fact that functions of Grassmann odd coordinates are necessarily polynomial, means that the weight vector fields on non-negatively graded supermanifolds and the closely related $N$-manifolds are h-complete. One should also note that Ševera [41] takes as his definition of an $N$-manifold a supermanifold equipped with an action of $(\mathbb{R}, \cdot)$ such that $-1 \in \mathbb{R}$ acts as the parity operator (it flips sign of any Grassmann odd coordinate). Note however, that Ševera does not offer a proof that homogeneous local coordinates can always be found and so it is not immediately clear if his notion of an $N$-manifold exactly coincides with that of Roytenberg. This equivalence follows from minor modification of the arguments made in the previous remark.

A graded bundle of degree $k$ admits a sequence of polynomial fibrations, where a point of $F_{l}$ is a class of the points of $F$ described in an affine coordinate system by the coordinates of weight $\leq l$, with the obvious tower of surjections

$$
F=F_{k} \stackrel{\tau^{k}}{\longrightarrow} F_{k-1} \stackrel{\tau^{k-1}}{\longrightarrow} \cdots \stackrel{\tau^{3}}{\longrightarrow} F_{2} \stackrel{\tau^{2}}{\longrightarrow} F_{1} \stackrel{\tau^{1}}{\longrightarrow} F_{0}=M
$$

where the coordinates on $M$ have zero weight. Note that $F_{1} \rightarrow M$ is a linear fibration and the other fibrations $F_{l} \rightarrow F_{l-1}$ are affine fibrations in the sense that the changes of local coordinates for the fibres are linear plus and additional additive terms of appropriate weight. The model fibres here are $\mathbb{R}^{N}$ (cf. [15]). We will also use on occasion $\tau:=\tau_{0}^{k}: F_{k} \rightarrow M$.

Canonical examples of graded bundles are, for instance, vector bundles, $n$-tuple vector bundles, higher tangent bundles $\mathrm{T}^{k} M$, and multivector bundles $\wedge^{n} \mathrm{~T} E$ of vector bundles $\tau: E \rightarrow M$ with respect to the projection $\wedge^{n} \mathrm{~T} \tau: \wedge^{n} \mathrm{~T} E \rightarrow \wedge^{n} \mathrm{~T} M$ (see [13]). If the weight is constrained to 
be either zero or one, then the weight vector field is precisely a vector bundle structure on $F$ and will be generally referred to as an Euler vector field.

The notion of a double vector bundle [37] (or a higher $n$-tuple vector bundle) is conceptually clear in the graded language in terms of mutually commuting homogeneity structures, or equivalently weight vector fields; see [14, 15]. This leads to the higher analogues known as $n$-tuple graded bundles, which are manifolds for which the structure sheaf carries an $\mathbb{N}^{n}$-grading such that all the weight vector fields are h-complete and pairwise commuting. In particular a double graded bundle consists of a manifold and a pair of mutually commuting and h-complete weight vector fields. If all the weights are either zero or one, then we speak of an $n$-tuple vector bundle.

\section{$2.2 \quad Q$-manifolds and related structures}

Let us briefly define some structures on supermanifolds that we will employ throughout this paper. The main purpose is to set some nomenclature and notation. The graded versions will be fundamental throughout this paper.

Definition 2.4. An odd vector field $Q \in \operatorname{Vect}(\mathcal{M})$ on a supermanifold $\mathcal{M}$ is said to be a homological vector field if and only if $2 Q^{2}=[Q, Q]=0$. Note that, as we have an odd vector field, this condition is generally non-trivial. A pair $(\mathcal{M}, Q)$, where $\mathcal{M}$ is a supermanifold and $Q \in \operatorname{Vect}(\mathcal{M})$ is called a $Q$-manifold. A morphism of $Q$-manifolds is a morphism of supermanifolds that relates the respective homological vector fields.

The nomenclature ' $Q$-manifold' is due to Schwarz [40]. As is well known, $Q$-manifolds, equipped with an additional grading, give a very economical description of Lie algebroids as first discovered by Vaŭntrob [43]. Recall the standard notion of a Lie algebroid as a vector bundle $E \rightarrow M$ equipped with a Lie bracket on the sections $[\bullet, \bullet]: \operatorname{Sec}(E) \times \operatorname{Sec}(E) \rightarrow \operatorname{Sec}(E)$ together with an anchor $\rho: \operatorname{Sec}(E) \rightarrow \operatorname{Vect}(M)$ that satisfy the Leibniz rule

$$
[u, f v]=\rho(u)[f] v+f[u, v]
$$

for all $u, v \in \operatorname{Sec}(E)$ and $f \in C^{\infty}(M)$. The Leibniz rule implies that the anchor is actually a Lie algebra morphism: $\rho([u, v])=[\rho(u), \rho(v)]$ (see, e.g., [11]). If we pick some local basis for the sections $\left(e_{a}\right)$, then the structure functions of a Lie algebroid are defined by

$$
\left[e_{a}, e_{b}\right]=C_{a b}^{c}(x) e_{c}, \quad \rho\left(e_{a}\right)=\rho_{a}^{A}(x) \frac{\partial}{\partial x^{A}},
$$

where we have local coordinates $\left(x^{A}\right)$ on $M$. These structure functions satisfy some compatibility conditions which can be neatly encoded in a homological vector field of weight one on $\Pi E$. Let us employ local coordinates

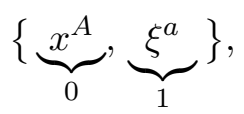

on $\Pi E$. Here the weight of coordinates corresponds to the natural weight induced by the homogeneity structure associated with the vector bundle structure [14] and this also corresponds to the Grassmann parity. We just briefly remark that one can also define Lie algebroids in the category of supermanifolds where it natural to consider the weight and Grassmann parity as being independent. The homological vector field encoding the Lie algebroid is

$$
Q=\xi^{a} \rho_{a}^{A}(x) \frac{\partial}{\partial x^{A}}+\frac{1}{2} \xi^{a} \xi^{b} C_{b a}^{c}(x) \frac{\partial}{\partial \xi^{c}} .
$$

The axioms of a Lie algebroid are then equivalent to $2 Q^{2}=[Q, Q]=0$. We will, by minor abuse of nomenclature, also refer to the graded $Q$-manifold $(\Pi E, Q)$ as a Lie algebroid. 
A morphism of Lie algebroid is then understood as a morphism of graded $Q$-manifolds. That is, we have a morphism of super graded bundles that relates the respective homological vector fields. Note that the definition of a Lie algebroid morphism in terms of $Q$-manifold morphisms is equivalent to the less obvious notion of a morphism of Lie algebroids as described in [28].

Example 2.5. Any Lie algebra $(\mathfrak{g},[]$,$) can be encoded in a homological vector field on the$ linear supermanifold $\Pi \mathfrak{g}$. Let us employ local coordinates $\left(\xi^{a}\right)$ on $\Pi \mathfrak{g}$; then we have

$$
Q=\frac{1}{2} \xi^{a} \xi^{b} C_{b a}^{c} \frac{\partial}{\partial \xi^{c}}
$$

where $C_{b a}^{c}$ is the structure constant of the Lie algebra in question. In this case the base manifold is just a point and the anchor map is trivial.

Example 2.6. In the other extreme, the tangent bundle of a manifold $T M$ is naturally a Lie algebroid for which the anchor is the identity map. The homological vector on ПТ $M$ that encodes the Lie algebroid structure is nothing other than the de Rham differential.

Definition 2.7. An odd Hamiltionain $S \in C^{\infty}\left(\mathrm{T}^{*} \mathcal{M}\right)$ that is quadratic in momenta (i.e., fibre coordinates) is said to be a Schouten structure if and only if $\{S, S\}=0$, where the bracket is the canonical Poisson bracket on the cotangent bundle. A pair $(\mathcal{M}, S)$, where $\mathcal{M}$ is a supermanifold and $S \in C^{\infty}\left(\mathrm{T}^{*} \mathcal{M}\right)$ is a Schouten structure, is called a $S$-manifold. The associated Schouten bracket on $C^{\infty}(M)$ is given as a derived bracket

$$
\llbracket f, g \rrbracket_{S}:=\{\{f, S\}, g\} .
$$

We must remark here that non-trivial Schouten structures only exist on supermanifolds. A Schouten bracket is also known as an odd Poisson bracket and satisfies the appropriate graded versions of the Jacobi identity and Leibniz rule.

Example 2.8. A Lie algebroid structure on a vector bundle $E \rightarrow M$ can be encoded in a weight one Schouten structure on the supermanifold $\mathrm{T}^{*} \Pi E^{*}$. Let us employ natural local coordinates on $\mathrm{T}^{*} \Pi E^{*}$ (with indicated bi-degrees)

$$
(\underbrace{x^{A}}_{(0,0)}, \underbrace{\chi_{a}}_{(0,1)}, \underbrace{p_{B}}_{(1,1)}, \underbrace{\theta^{b}}_{(1,0)})
$$

Then, the Schouten structure encoding a Lie algebroid is given by

$$
S=\theta^{a} \rho_{a}^{A}(x) p_{A}+\frac{1}{2} \theta^{a} \theta^{b} C_{b a}^{c}(x) \chi_{c}
$$

and the axioms of a Lie algebroid are equivalent to $\{S, S\}=0$, where the bracket here is the canonical Poisson bracket on the cotangent bundle.

Definition 2.9. A supermanifold $M$ is said to be a $Q S$-manifold if it is simultaneously a $Q$ manifold and a $S$-manifold such that $L_{Q} S:=\{\mathcal{Q}, S\}=0$, where $\mathcal{Q} \in C^{\infty}\left(\mathrm{T}^{*} M\right)$ is the symbol of the homological vector field $Q$.

As the symbol map sends the Lie bracket of vector fields to the Poisson bracket, a $Q S$ manifold can be considered as a supermanifold equipped with odd Hamiltonians, one linear and one quadratic in momenta, that satisfy $\{\mathcal{Q}, \mathcal{Q}\}=0,\{S, S\}=0$ and $\{\mathcal{Q}, S\}=0$. The definition of a $Q S$-manifold goes back to Voronov [44].

Graded $Q S$-manifolds give us a convenient way to understand Lie bi-algebroids, which is due to Voronov [44], but also see Kosmann-Schwarzbach [22] who modified the original definition of 
Mackenzie and $\mathrm{Xu}$ [29]. The original definition involved the differential associated with the dual Lie algebroid and Lie bracket on sections of the Lie algebroid, and not the associated Schouten bracket on 'multivector fields'. Following Kosmann-Schwarzbach, a Lie bi-algebroid is a pair of Lie algebroids $\left(E, E^{*}\right)$ such that

$$
Q_{E} \llbracket X, Y \rrbracket_{E^{*}}=\llbracket Q_{E} X, Y \rrbracket_{E^{*}}+(-1)^{\widetilde{X}} \llbracket X, Q_{E} Y \rrbracket_{E^{*}},
$$

for all 'multivector fields' $X$ and $Y \in C^{\infty}(\Pi E)$. That is the homological vector field encoding the Lie algebroid structure on $E$ must be a derivation with respect to the Schouten bracket that encodes the Lie algebroid structure on the dual vector bundle $E^{*}$. It is not hard to see that this definition is equivalent to the compatibility condition

$$
\mathcal{L}_{Q_{E}} S_{E^{*}}=\left\{\mathcal{Q}_{E}, S_{E^{*}}\right\}=0
$$

and so we have a $Q S$-manifold. Here we use the canonical isomorphism $\mathrm{T}^{*} \Pi E \simeq \mathrm{T}^{*} \Pi E^{*}$. In natural local coordinates the symbol of the homological vector field and the Schouten structure are given by

$$
\mathcal{Q}_{E}=\theta^{a} \rho_{a}^{A}(x) p_{A}+\frac{1}{2} \theta^{a} \theta^{b} C_{b a}^{c}(x) \chi_{c}, \quad S_{E^{*}}=\chi_{a} \rho^{a A}(x) p_{A}+\frac{1}{2} \chi_{a} \chi_{b} C_{c}^{b a}(x) \theta^{c},
$$

which are clearly of bi-weight $(2,1)$ and $(1,2)$ respectively. We can then define a Lie bi-algebroid as the graded $Q S$-manifold ( $\left.\mathrm{T}^{*} \Pi E, Q_{E}, S_{E^{*}}\right)$.

The above definition of a Lie bi-algebroid is not manifestly symmetric in $E$ and $E^{*}$, however, due to the isomorphism

$$
\left(\mathrm{T}^{*} \Pi E, Q_{E}, S_{E^{*}}\right) \simeq\left(\mathrm{T}^{*} \Pi E^{*}, S_{E}, Q_{E^{*}}\right),
$$

it is clear that if $\left(E, E^{*}\right)$ is a Lie bi-algebroid, then so is $\left(E^{*}, E\right)$.

\subsection{Weighted Lie algebroids}

One can think of a weighted Lie algebroid as a Lie algebroid in the category of graded bundles or, equivalently, as a graded bundle in the category of Lie algebroids [3]. Thus one should think of weighted Lie algebroids as Lie algebroids that carry an additional compatible grading. For the purposes of this paper, we will define weighted Lie algebroids here using the notion of homogeneity structures as this will turn out to be a useful point of view when dealing with the associated Lie theory. Let us recall the definition of a graded-linear bundle, which is fundamental in the notion of a weighted Lie algebroid.

Definition 2.10. A manifold $D_{(k-1,1)}$ equipped with a pair of homogeneity structures $(\widehat{\mathrm{h}}, \widehat{\mathrm{l}})$ of degree $k-1$ and 1 respectively is called a graded-linear bundle of degree $k$, which we will abbreviate as $\mathcal{G} \mathcal{L}$-bundle, if and only if the respective actions commute.

The above definition is equivalent to the definition given in [3] in terms of mutually commuting h-complete weight vector fields of degree $k-1$ and 1 . In all, by passing to total weight, a $\mathcal{G L}$-bundle is a graded bundle of degree $k$. We will denote the base defined by the vector bundle structure as $B_{k-1} \rightarrow M$. We will generally employ the shorthand notation $D_{k}$ for a $\mathcal{G L}$-bundle $D_{(k-1,1)}$ of degree $k$ in this paper.

Example 2.11 ([3]). Let $F_{k-1}$ be a graded bundle of degree $k-1$, then $\mathrm{T} F_{k-1}$ is canonically a $\mathcal{G L}$-bundle of degree $k$. The degree $k-1$ homogeneity structure is simply the tangent lift of the homogeneity structure on the initial graded bundle, while the degree one homogeneity structure is that associated with the natural vector bundle structure of the tangent bundle. That is, if we 
equip $F_{k-1}$ with local coordinates $\left(x_{u}^{\alpha}\right)$, here $0 \leq u<k$, then $\mathrm{T} F_{k-1}$ can be naturally equipped with coordinates

$$
(\underbrace{x_{u}^{\alpha}}_{(u, 0)}, \underbrace{\delta x_{u+1}^{\beta}}_{(u, 1)}) .
$$

Example 2.12 ([3]). Similarly to the above example, if $F_{k-1}$ be a graded bundle of degree $k-1$, then $\mathrm{T}^{*} F_{k-1}$ is canonically a $\mathcal{G} \mathcal{L}$-bundle of degree $k$. Note there we have to employ the phase lift of the homogeneity structure on $F_{k-1}$ to ensure that we do not leave the category of graded bundles [12]; that is we do not want negative weight coordinates. This amounts to an appropriate shift in the weight and allows us to employ homogeneous local coordinates of the form

$$
(\underbrace{x_{u}^{\alpha}}_{(u, 0)}, \underbrace{p_{\beta}^{k-u}}_{(k-1-u, 1)}) .
$$

Note that as we have a linear structure on $\mathcal{G} \mathcal{L}$-bundles, that is we have a homogeneity structure of weight one, or equivalently an Euler vector field, applying the parity reversion functor makes sense. Thus, we can consider the $\mathcal{G L}$-antibundle $\left(\Pi D_{k}, \Pi \widehat{\mathrm{h}}, \Pi \widehat{\mathrm{l}}\right)$, where we define viz

$$
\Pi \widehat{h}_{t}: \Pi D_{k} \rightarrow \Pi D_{k}
$$

and similarly for $\widehat{\Pi 1}$. We can now define a weighted Lie algebroid as follows;

Definition 2.13. A weighted Lie algebroid of degree $k$ is the quadruple

$$
\left(\Pi D_{k}, \Pi \widehat{\mathrm{h}}, \widehat{\Pi \mathrm{l}}, Q\right) \text {, }
$$

where $\left(\Pi D_{k}, Q\right)$ is a $Q$-manifold and $\left(\Pi D_{k}, \Pi \widehat{\mathrm{h}}, \widehat{\Pi \mathrm{l}}\right)$ is a $\mathcal{G L}$-antibundle such that

$$
\begin{aligned}
& Q \circ\left(\Pi \widehat{\mathrm{h}}_{t}\right)^{*}=\left(\Pi \widehat{\mathrm{h}}_{t}\right)^{*} \circ Q, \\
& s Q \circ\left(\Pi \widehat{\Pi l}_{s}\right)^{*}=\left(\Pi \widehat{\Pi l}_{s}\right)^{*} \circ Q,
\end{aligned}
$$

for all $t$ and all $s \in \mathbb{R}$.

The above definition is really just the statement that a weighted Lie algebroid can be defined as a $\mathcal{G L}$-antibundle equipped with a homological vector of bi-weight $(0,1)$. By passing to total weight, weighted Lie algebroids produce examples of higher Lie algebroids, cf. Voronov [45].

Example 2.14. It is not hard to see that a weighted Lie algebroid of degree 1 is just a standard Lie algebroid; the additional homogeneity structure is trivial and so we have a graded super bundle of degree one and a weight one homological vector field.

Example 2.15. Similarly to the previous example, weighted Lie algebroids of degree 2 (i.e., of bi-degree $(1,1))$ are $\mathcal{V B}$-algebroids; we now have a double super vector bundle structure and a weight $(0,1)$ homological vector field [16].

Further natural examples of weighted Lie algebroids include the tangent bundle of a graded bundle and the higher order tangent bundles of a Lie algebroid, see [3].

The above definition allows for a further more economical definition via the following proposition, which follows immediately from (2.3). 
Proposition 2.16. A weighted Lie algebroid of degree $k$ can equivalently be defined as a Lie algebroid $(\Pi E, Q)$ equipped with a homogeneity structure of degree $k-1$ such that

$$
\Pi \widehat{\mathrm{h}}_{t}: \Pi E \rightarrow \Pi E
$$

is a Lie algebroid morphism for all $t \in \mathbb{R}$.

Remark 2.17. Bursztyn, Cabrera and del Hoyo [5, Theorem 3.4.3] establish that $\mathcal{V B}$-algebroids can be defined as Lie algebroids equipped with a compatible regular homogeneity structure. Note that they make explicit use of the Poisson structure associated with a Lie algebroid, where we prefer the (equivalent) description in terms of $Q$-manifolds. Their motivation for describing $\mathcal{V B}$-algebroids in terms of compatible homogeneity structures is, much like our motivation, to tackle the associated Lie theory.

To get a more traditional description of a weighted Lie algebroid, let us employ homogeneous local coordinates

$$
(\underbrace{x_{u}^{\alpha}}_{(u, 0)}, \underbrace{\theta_{u+1}^{I}}_{(u, 1)}),
$$

on $\Pi D_{k}$, where $0 \leq u \leq k-1$ and this label refers to the total weight. Note that the second component of the bi-weight encodes the Grassmann parity of the coordinates; that is the $\theta$ 's are anticommuting coordinates. In these homogeneous local coordinates the homological vector field encoding the structure of a weighted Lie algebroid is

$$
Q=\theta_{u-u^{\prime}+1}^{I} Q_{I}^{\alpha}\left[u^{\prime}\right](x) \frac{\partial}{\partial x_{u}^{\alpha}}+\frac{1}{2} \theta_{u^{\prime \prime}-u^{\prime}+1}^{I} \theta_{u-u^{\prime \prime}+1}^{J} Q_{J I}^{K}\left[u^{\prime}\right](x) \frac{\partial}{\partial \theta_{u+1}^{K}},
$$

where $Q_{I}^{\alpha}\left[u^{\prime}\right]$ and $Q_{I J}^{K}\left[u^{\prime}\right]$ are the homogenous parts of the structure functions of degree $u^{\prime}$. In the notation employed here, any $\theta$ with seemingly zero or negative total weight are set to zero.

In [3] homogeneous linear sections of $D_{k} \rightarrow B_{k-1}$ of weight $r$ were defined functions on the $\mathcal{G L}$-bundle $D_{k}^{*}$ of bi-weight $(r-1,1)$. Let us equip $D_{k}^{*}$ with homogeneous local coordinates

$$
(\underbrace{x_{u}^{\alpha}}_{(u, 0)}, \underbrace{\pi_{I}^{u+1}}_{(u, 1)}) .
$$

In these local coordinates a linear section of $D_{k}$ is given by

$$
s_{r}=s_{r-u-1}^{I}(x) \pi_{I}^{u+1} .
$$

Such linear sections can also be understood as vertical vector fields constant along the fibres of $\Pi D_{k}$. In the graded language we consider vector fields that are of weight -1 with respect to the second component of the bi-weight on $\Pi D_{k}$. Note that we have a shift in the bi-weight and also the Grassmann parity. The reason we use $\Pi D_{k}$ rather than $D_{k}$ will become clear in a moment when we consider how to construct the Lie algebroid bracket and the anchor. By employing the homogeneous local coordinates on $\Pi D_{k}$ introduced perviously we have the identification

$$
s_{r} \leftrightarrow i_{s_{r}}:=s_{r-u-1}^{I}(x) \frac{\partial}{\partial \theta_{k-u}^{I}},
$$

which is really no more than the 'interior product' generalised to sections of a vector bundle. We do not require any extra structure here for this identification, just the vector bundle structure 
is required. Note the shift in the bi-weight $(r-1,1) \rightarrow(r-k,-1)$, or in other words we have a shift of $(-(k-1),-2)$. This shift will be very important.

We can now employ the derived bracket formalism [23] to construct the Lie algebroid bracket;

$$
i_{\left[s_{1}, s_{2}\right]}=\left[\left[Q, i_{s_{1}}\right], i_{s_{2}}\right],
$$

up to a possible overall minus sign by convention. By inspection we see that $i_{\left[s_{1}, s_{2}\right]}$ is of bi-weight $\left(r_{1}+r_{2}-2 k,-1\right)$, where $s_{1}$ is a linear section of degree $r_{1}$ and similarly for $s_{2}$. This means that the Lie algebroid bracket $\left[s_{1}, s_{2}\right]$ is of bi-weight $\left(r_{1}+r_{2}-1-k, 1\right)$; that is the Lie algebroid bracket carries weight $-k$.

Definition 2.18. A morphism of weighted Lie algebroids is a morphism of the underlying Lie algebroids (understood as a morphism of $Q$-manifolds) that intertwines the respective additional homogeneity structures.

In particular, there is a forgetful functor from the category of weighted Lie algebroids to Lie algebroids given by forgetting the homogeneity structure. The image of this forgetful functor is not a full subcategory of the category of Lie algebroids. We will denote the category of Lie algebroids as Algd and the subcategory of weighted Lie algebroids as wAlgd.

Proposition 2.19. If $\left(\Pi D_{k}, Q\right)$ is weighted Lie algebroid of degree $k$, then each $\Pi D_{j}$ for $0 \leq$ $j<k$ comes with the structure of a weighted Lie algebroid of degree $j$. In particular, $\Pi E=\Pi D_{1}$ is a 'genuine' Lie algebroid. Moreover, the projection form any 'higher level' to a 'lower level' is a morphism of weighted Lie algebroids.

Proof. Because of the condition that the weight of $Q$ is $(0,1)$ and that there are no negatively graded coordinates, we know that $Q$ is projectable to any of the 'levels' of the affine fibrations

$$
\Pi D_{(k-1,1)} \rightarrow \Pi D_{(k-2,1)} \rightarrow \cdots \rightarrow \Pi D_{(1,1)} \rightarrow \Pi D_{(0,1)} .
$$

This can be directly checked using the local expression for the homological vector field. Moreover, the projection of $Q$ to any level is a homological vector field of weight $(0,1)$ and hence we have the structure of a weighted Lie algebroid. The fact that the projections give rise to weighted Lie algebroid morphisms follows directly.

\subsection{Lie groupoids}

Here we shall set some notation and recall some well-known results. Let $\mathcal{G} \rightrightarrows M$ be an arbitrary Lie groupoid with source map $\underline{s}: \mathcal{G} \rightarrow M$ and target map $\underline{t}: \mathcal{G} \rightarrow M$. There is also the inclusion map $\iota: M \rightarrow \mathcal{G}$ and a partial multiplication $(g, h) \mapsto g \cdot h$ which is defined on $\mathcal{G}^{(2)}=\{(g, h) \in$ $\mathcal{G} \times \mathcal{G}: \underline{s}(g)=\underline{t}(h)\}$. Moreover, the manifold $\mathcal{G}$ is foliated by $\underline{s}$-fibres $\mathcal{G}_{x}=\{g \in \mathcal{G} \mid \underline{s}(g)=x\}$, where $x \in M$. As by definition the source and target maps are submersions, the $\underline{s}$-fibres are themselves smooth manifolds. Geometric objects associated with this foliation will be given the superscript $\underline{s}$. In particular, the distribution tangent to the leaves of the foliation will be denoted by $\mathrm{T}^{\underline{s}} \mathcal{G}$. To ensure no misunderstanding with the notion of a Lie groupoid morphism we recall the definition we will be using.

Definition 2.20. Let $\mathcal{G}_{i} \rightrightarrows M_{i}(i=1,2)$ be a pair of Lie groupoids. Then a Lie groupoid morphisms is a pair of maps $(\Phi, \phi)$ such that the following diagram is commutative

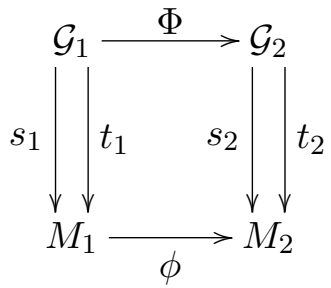


in the sense that $\underline{s}_{2} \circ \Phi=\phi \circ \underline{s}_{1}$ and $\underline{t}_{2} \circ \Phi=\phi \circ \underline{t}_{1}$, subject to the further condition that $\Phi$ respect the (partial) multiplication; if $g, h \in \mathcal{G}_{1}$ are composable, then $\Phi(g \cdot h)=\Phi(g) \cdot \Phi(h)$. It then follows that for $x \in M_{1}$ we have $\Phi\left(\mathbb{1}_{x}\right)=\mathbb{1}_{\phi(x)}$ and $\Phi\left(g^{-1}\right)=\Phi(g)^{-1}$.

We will denote the category of Lie groupoids as Grpd. It is well known that via a differentiation procedure one can construct the Lie functor

$$
\operatorname{Grpd} \stackrel{\text { Lie }}{\longrightarrow} \text { Algd, }
$$

that sends a Lie groupoid to its Lie algebroid, and sends morphisms of Lie groupoids to morphisms of the corresponding Lie algebroids. However, as is also well known, we do not have an equivalence of categories as not all Lie algebroids arise as the infinitesimal versions of Lie groupoids. There is no direct generalisation of Lie III, apart from the local case. The obstruction to the integrability of Lie algebroids, the so called monodromy groups, were first uncovered by Crainic and Fernandes [7]. For the transitive case the topological obstruction was uncovered by Mackenzie [26]. The interested reader can consult the original literature or the very accessible lecture notes also by Crainic and Fernandes [8]. To set some notation and nomenclature, given a Lie groupoid $\mathcal{G}$, we say that $\operatorname{Lie}(\mathcal{G})=\mathrm{A}(\mathcal{G})$ integrates $\mathrm{A}(\mathcal{G})$. Moreover, if $\Phi: \mathcal{G} \rightarrow \mathcal{H}$ is a morphism of Lie groupoids, then we will write $\Phi^{\prime}=\operatorname{Lie}(\Phi): \mathrm{A}(\mathcal{G}) \rightarrow \mathrm{A}(\mathcal{H})$ for the corresponding Lie algebroid morphism, which actually comes from the differential $\mathrm{T} \Phi: \mathrm{T} \mathcal{G} \rightarrow \mathrm{T} \mathcal{H}$ restricted to $\underline{s}$-fibres at the submanifold $M$.

Let us just recall Lie II theorem, as we will need it later on.

Theorem 2.21 (Lie II). Let $\mathcal{G} \rightrightarrows M$ and $\mathcal{H} \rightrightarrows N$ be Lie groupoids. Suppose that $\mathcal{G}$ is source simply-connected and that $\phi: \mathrm{A}(\mathcal{G}) \rightarrow \mathrm{A}(\mathcal{H})$ is a Lie algebroid morphism between the associated Lie algebroids. Then, $\phi$ integrates to a unique Lie groupoid morphisms $\Phi: \mathcal{G} \rightarrow \mathcal{H}$ such that $\Phi^{\prime}=\phi$.

This generalisation of Lie II to the groupoid case was first proved by Mackenzie ans Xu [30]. A simplified proof was obtained shortly after by Moerdijk and Mrčun [36]. Note that the assumption that the Lie groupoid $\mathcal{G}$ must be source simply-connected is essential.

\section{$3 \quad$ Weighted Lie groupoids}

To avoid any possible confusion with the overused adjective 'graded', following the nomenclature in [3] we will refer to weighted rather than graded Lie groupoids. The term 'graded groupoid' appears in the work of Mehta [33] as groupoids in the category of $\mathbb{Z}$-graded manifolds; we will comment on this further. In this work we will content ourselves with working in the strictly commutative (in opposition to graded-commutative) setting. The basic idea is to take the definition of a Lie groupoid and replace 'smooth manifold' everywhere with 'graded bundle'. To do this economically, we will make use of the description of graded bundles in terms of smooth manifolds equipped with a homogeneity structure of degree $k$, so that morphisms of graded bundles are just smooth maps intertwining the respective homogeneity structures. In essence, we require that the Lie groupoid structure functions respect the graded structure which is encoded in the homogeneity structure.

\subsection{The definition of weighted Lie groupoids via homogeneity structures}

Let us formalise the comments in the beginning of this section with the following definition;

Definition 3.1. A weighted Lie groupoid of degree $k$ is a Lie groupoid $\Gamma_{k} \rightrightarrows B_{k}$, together with a homogeneity structure $\underline{\mathrm{h}}: \mathbb{R} \times \Gamma_{k} \rightarrow \Gamma_{k}$ of degree $k$, such that $\underline{\mathrm{h}}_{t}$ is a Lie groupoid morphism 
for all $t \in \mathbb{R}$. Such homogeneity structures will be referred to as multiplicative homogeneity structures.

Remark 3.2. Equivalently one could think of a weighted Lie groupoid in terms of multiplicative weight vector fields. This follows from the definition of a weighted Lie groupoid and the fact that weight vector fields associated with homogeneity structures are complete. However, it will be convenient to use the homogeneity structures rather than weight vector fields from the perspective of Lie theory.

Let us unravel some of the structure here. First, as both the source and target maps are submersions and by assumption $\Gamma_{k}$ is a graded bundle of degree $k, B_{k}$ is also a graded bundle of degree $k$ (we will consider lower degree graded bundles to be included as higher degree graded bundles if necessary). Secondly, it will be convenient to consider the homogeneity structure $\underline{h}$ as a pair of structures: $\underline{\mathrm{h}}_{t}=\left(\mathrm{h}_{t}, \mathrm{~g}_{t}\right)$. Then, consider the following commutative diagram

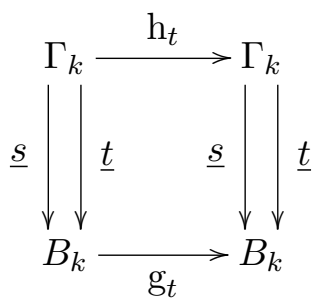

which means that $\underline{s} \circ \mathrm{h}_{t}=\mathrm{g}_{t} \circ \underline{s}$, and $\underline{t} \circ \mathrm{h}_{t}=\mathrm{g}_{t} \circ \underline{t}$. That is, the source and target maps intertwine the respective homogeneity structure. Furthermore, we have $\mathrm{h}_{t}(g \cdot h)=\mathrm{h}_{t}(g) \cdot \mathrm{h}_{t}(h)$, meaning that the homogeneity structure respects the (partial) multiplication, or indeed vice versa. From the definition of a Lie groupoid morphism, compatibility with the identities and inverses follows. In short, the graded structure is fully compatible with the Lie groupoid structure. Or, in other words, we have a Lie groupoid object in the category of graded bundles or equivalently vice-versa.

Definition 3.3. A morphism of weighted Lie groupoids is a Lie groupoid morphism that intertwines the respective homogeneity structures.

In other words, a morphism of weighted Lie groupoids must in addition to being a morphism of Lie groupoids be a morphism of graded bundles. Clearly we have a forgetful functor from the category of weighted Lie groupoids to the category of Lie groupoids which forgets the homogeneity structure. Similar to the case of algebroids, the image of this forgetful functor is not a full subcategory of the category of Lie groupoids. We will denote the category of weighted Lie groupoids as wGrpd.

Remark 3.4. Graded groupoids in the sense of Mehta [33, 34] are understood as groupoid objects in the category of $\mathbb{Z}$-graded manifolds. The $\mathbb{Z}_{2}$-grading is a consequence of the $\mathbb{Z}$-grading and so $\mathbb{Z}$-manifolds are not quite as general as Voronov's graded supermanifolds (cf. [44]). Without details, a graded groupoid is a pair of $\mathbb{Z}$-graded manifolds $(\mathcal{G}, M)$, equipped with surjective submersions $s, t: \mathcal{G} \rightrightarrows M$ (the source and target), together with a partial multiplication map, an identity map and an inverse map which all satisfy the appropriate commutative diagrams to define a groupoid structure. To be very clear, all the structural maps are morphisms in the category of $\mathbb{Z}$-graded manifolds. One should of course be reminded of the definition of Lie supergroups as group objects in the category of supermanifolds and indeed Mehta's constructions cover the case of Lie supergroupoids.

From the definition of a weighted Lie groupoid one can check directly that all the structural maps are morphisms in the category of graded bundles; all the maps relate the respective weight vector fields (or equivalently the actions of the respective homogeneity structures). Thus, one could start with a definition of a weighted Lie groupoid in terms closer to that used by Mehta 
to define his graded groupoids. However, in light of Lie theory it will turn out to be very convenient to describe weighted Lie groupoids as Lie groupoids with a compatible homogeneity structure. Mehta by working in the category of $\mathbb{Z}$-graded manifolds did not have anything like a homogeneity structure at his disposal. While it is straight forward to show that differentiating graded groupoids leads to graded algebroids, the question of integration seems less tractable.

\subsection{Relation with $\mathcal{V} \mathcal{B}$-groupoids}

Bursztyn, Cabrera and del Hoyo (cf. [5, Theorem 3.2.3]) establish a one-to-one correspondence between $\mathcal{V B}$-groupoids, which have several equivalent definitions (see for example [17]), and Lie groupoids that have a regular action of $(\mathbb{R}, \cdot)$ as Lie groupoid morphisms. In essence, they show the equivalence between $\mathcal{V B}$-groupoids and weighted Lie groupoids of degree one. We take the attitude that the 'correct definition' of a $\mathcal{V B}$-groupoid is implied by this correspondence. We consider the double structure, that is the pair of Lie groupoids and the pair of vector bundles together with a collection of not-so obvious compatibility conditions, as being derived rather than fundamental. This helps motivate our definition of weighted Lie groupoids using homogeneity structures.

Proposition 3.5. For any weighted Lie groupoid $\Gamma_{k} \rightrightarrows B_{k}$ there is an underlying 'genuine' or 'ungraded' Lie groupoid $\mathcal{G} \rightrightarrows M$ described by the following surjective groupoid morphism

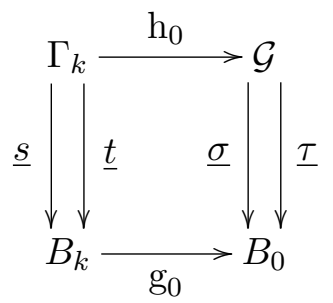

Moreover, if $\Gamma_{k}$ is source simply-connected, then so is $\mathcal{G}$.

Proof. By construction we have projections $\mathrm{h}_{0}: \Gamma_{k} \rightarrow \Gamma_{0}:=\mathcal{G}$ and $\mathrm{g}_{0}: B_{k} \rightarrow B_{0}:=M$. Thus $\mathcal{G} \subset \Gamma_{k}$ and $M \subset \mathcal{G}$ are embedded manifolds. Clearly $\mathcal{G}$ and $M$ define a (set theoretical) subgroupoid of $\Gamma_{k} \rightrightarrows B_{k}$. As $\left(\mathrm{h}_{t}, \mathrm{~g}_{t}\right)$ is a morphism of Lie groupoids for all $t \in \mathbb{R}$, including zero, it follows that the source map $\underline{\sigma}: \mathcal{G} \rightarrow M$ is a submersion. Thus we have the structure of a Lie groupoid. Now consider any point $p \in \mathcal{G}$. As $\mathrm{h}_{0}(p)=p$ the we have an induced retraction map $\underline{s}^{-1}(p) \rightarrow \underline{\sigma}^{-1}(p)$ and thus we have a homomorphisms between the respective fundamental groups. Thus if $\Gamma_{k}$ is source simply-connected, then so is $\mathcal{G}$.

Actually, slightly modifying the above proof, we can derive the groupoid version of the tower of Lie algebroid structures (2.4).

Proposition 3.6. If $\Gamma_{k} \rightrightarrows B_{k}$ is a weighted Lie groupoid of degree $k$, then we have the following tower of weighted groupoid structures of lower order and their morphisms:

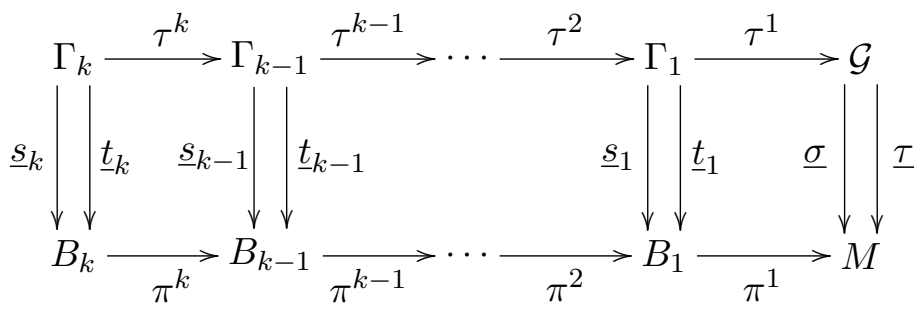

In particular, $\Gamma_{1} \rightrightarrows B_{1}$ is a $\mathcal{V B}$-groupoid. 


\subsection{Examples of weighted Lie groupoids}

In this subsection we sketch some simple examples of weighted Lie groupoids following adaptation of some of the standard examples of Lie groupoids and graded bundles. We expect more intricate examples to follow, some of which maybe more useful in applications.

Example 3.7. All graded bundles can be considered as weighted Lie groupoids over themselves by taking all the structure maps to be the identity. This leads to the notion of a weighted unit Lie groupoid.

Example 3.8. Just as Lie groups are examples of Lie groupoids (over a single point), we can consider weighted Lie groups of degree $k$ which are Lie groups $G_{k}$ equipped with a compatible homogeneity structure of degree $k$. The compatibility condition is simply $\mathrm{h}_{t}(g \cdot h)=\mathrm{h}_{t}(g) \cdot \mathrm{h}_{t}(h)$ for all elements $g, h \in G_{k}$.

Example 3.9. If $F_{k} \rightarrow M$ is a graded bundle of degree $k$, then we can construct the weighted pair groupoid in the obvious way; $F_{k} \times F_{k} \rightrightarrows F_{k}$. Underlying this is the 'genuine' pair groupoid $M \times M \rightrightarrows M$. Moreover, as we have a series of affine fibrations associated with any graded bundle the weighted pair groupoid can be 'fibred' as $F_{k} \times_{F_{q}} F_{k} \rightrightarrows F_{k}$ in the obvious way.

Example 3.10. Let $\mathcal{G} \rightrightarrows M$ be a Lie groupoid. Then associated with this is the higher tangent Lie groupoid $\mathrm{T}^{k} \mathcal{G} \rightrightarrows \mathrm{T}^{k} M$, which is naturally a weighted Lie groupoid and is formed by applying the higher tangent functor of order $k$ to the structure maps of $\mathcal{G} \rightrightarrows M$. Clearly the initial Lie groupoid is the 'genuine' Lie groupoid underlying the higher tangent Lie groupoid.

Example 3.11. Similar to the previous example, one can consider iterating the tangent functor $k$-times to construct the iterated tangent Lie groupoid $\mathrm{T}^{(k)} \mathcal{G} \rightrightarrows \mathrm{T}^{(k)} M$. By passing to total weight we have a weighted Lie groupoid of degree $k$.

Example 3.12. As already commented on, $\mathcal{V B}$-groupoids are weighted Lie groupoids of degree 1. Standard examples of $\mathcal{V B}$-groupoids include $\mathrm{T} \mathcal{G} \rightrightarrows \mathrm{T} M$ and $\mathrm{T}^{*} \mathcal{G} \rightrightarrows \mathrm{A}^{*}(\mathcal{G})$ for any Lie groupoid $\mathcal{G} \rightrightarrows M$.

Example 3.13. Combining Examples 3.10 and 3.12, we can consider the higher Pontryagin Lie groupoid

$$
\mathrm{T}^{k} \mathcal{G} \times \mathrm{G}^{*} \mathcal{G} \rightrightarrows \mathrm{T}^{k} M \times_{M} \mathrm{~A}^{*}(\mathcal{G})
$$

as a weighted Lie groupoid by passing from the natural bi-weight to total weight.

Example 3.14. Let $\left(G_{l}, \mathrm{~h}\right)$ be a weighted Lie group and $\left(F_{k}, \mathrm{~g}\right)$ be a graded bundle. We assume that $G_{l}$ acts on $F_{k}$. Then we can construct the weighted action Lie groupoid as follows; $G_{l} \times F_{k} \rightrightarrows F_{k}$, where the source and target maps are given by

$$
\underline{s}(g, x)=x, \quad \underline{t}(g, x)=g x,
$$

and the (partial) multiplication being

$$
(h, y) \cdot(g, x)=(h g, x) .
$$

We define $\underline{\mathrm{h}}:=(\mathrm{h}, \mathrm{g})$ as the homogeneity structure on the Lie groupoid. Compatibility with the source map and partial multiplication is automatic from the definition of a homogeneity structure, but compatibility with the target map requires the natural condition $\mathrm{g}_{t}(g x)=\left(\mathrm{h}_{t} g\right)\left(\mathrm{g}_{t} x\right)$. Thus we will define a weighted action Lie groupoid via the construction of an action Lie groupoid, with the addition of compatible homogeneity structures as described above. 
Example 3.15. Any $\mathcal{G L}$-bundle $\left(D_{k}, \widehat{\mathrm{h}}, \widehat{\mathrm{l}}\right)$ can be considered as a weighed Lie groupoid in the following way. The base is defined as $B_{k-1}:=\widehat{1}_{0}\left(D_{k}\right)$ and the source and target maps are set to the projection $\underline{s}=\underline{t}=\widehat{l}_{0}$. Thus, the $\underline{s}$-fibres have the structure of graded spaces, cf. [15] (i.e., graded bundles over a point). The partial multiplication is just the addition in the $\underline{s}$-fibres. The compatibility of the Lie groupoid and graded bundle structures follows directly from the definition of a $\mathcal{G} \mathcal{L}$-bundle.

Example 3.16. The fibred product

$$
\mathrm{T} \mathcal{G} \times_{\mathcal{G}} \mathrm{T} \mathcal{G} \times_{\mathcal{G}} \cdots \times_{\mathcal{G}} \mathrm{T} \mathcal{G} \rightrightarrows \mathrm{T} M \times_{M} \mathrm{~T} M \times_{M} \cdots \times_{M} \mathrm{~T} M
$$

where $\mathcal{G} \rightrightarrows M$ is an arbitrary Lie groupoid, can be considered as a weighted Lie groupoid in the following way. The natural homogeneity structure associated with the $i$-th factor $\mathrm{T} \mathcal{G} \rightrightarrows \mathrm{T} M$ can be shifted by $i \in \mathbb{N}$ and then, by passing to total weight, we have a weighted Lie groupoid of degree $k$, assuming we the fibred product consists of $k$ factors.

\section{Lie theory for weighted groupoids and algebroids}

We now turn our attention to the Lie theory relating weighted Lie groupoids and weighted Lie algebroids. The differentiation of a weighted Lie groupoid produces rather naturally a weighted Lie algebroid. One can use the standard construction here, just taking a little care with the weights. The integration of a weighted Lie algebroid to a weighted Lie groupoid also follows via the standard constructions, again taking care with the graded structure. We will not address the full question of global integrability, rather we will assume integrability as a Lie algebroid and show that the weighted structure is naturally inherited.

\subsection{Differentiation of weighted Lie groupoids}

It is clear that as a Lie groupoid a weighted Lie groupoid always admits a Lie algebroid associated with it following the classical constructions. The question is if the associated Lie algebroid is in fact a weighted Lie algebroid?

Theorem 4.1. If $\Gamma_{k} \rightrightarrows B_{k}$ is a weighted Lie groupoid of degree $k$ with respect to a homogeneity structure $h$ on $\Gamma_{k}$, then $\mathrm{A}\left(\Gamma_{k}\right) \rightarrow B_{k}$ is a weighted Lie algebroid of degree $k+1$ with respect to the homogeneity structure $\widehat{h}$ defined by

$$
\widehat{h}_{t}=\left(h_{t}\right)^{\prime}=\operatorname{Lie}\left(h_{t}\right) .
$$

Proof. We need only follow the classical constructions taking care of the weight as we proceed. In particular, we need only show that $\mathrm{A}\left(\Gamma_{k}\right)$ has the structure of a $\mathcal{G} \mathcal{L}$-bundle and that the corresponding Lie algebroid brackets are of weight $-(k+1)$.

1. As $\mathrm{A}\left(\Gamma_{k}\right):=\left.\operatorname{ker}(\mathrm{T} \underline{s})\right|_{\iota\left(B_{k}\right)}$, it is clear that as a subbundle of $\mathrm{T} \Gamma_{k}$ that we have the structure of a $\mathcal{G L}$-bundle. The degree $k$ homogeneity structure $\widehat{h}$ is inherited from the tangent lift of the homogeneity structure on $\Gamma_{k}$ and the degree 1 homogeneity structure is inherited from the natural one related to the vector bundle structure of tangent bundles. In other words, $\widehat{h}_{t}=\left(h_{t}\right)^{\prime}$ is the 'differential' of $h$.

2. Sections of weight $r$ are by our definition functions on $\mathrm{A}\left(\Gamma_{k}\right)^{*}$ of bi-weight $(r-1,1)$, see Section 2. Thus, associating sections with right invariant vector fields requires a shift in the weights of $(-k,-2)$, which comes from associating momenta with derivatives, i.e., use weighted principle symbols. Thus, the prolongation of a section of weight $r$ to a left invariant vector field is a vector field of bi-weight $(r-1-k,-1)$. Thus if we take the Lie bracket of two sections of weight $r_{1}$ and $r_{2}$ as right invariant vector fields the resulting will be a right invariant vector 
field of weight $\left(r_{1}+r_{2}-2-2 k,-1\right)$. Now upon restriction and 'shifting back' to functions on $\mathrm{A}\left(\Gamma_{k}\right)^{*}$, the resulting section is of bi-weight $\left(r_{1}+r_{2}-1-(k+1), 1\right)$. Thus the Lie algebroid bracket carries weight $-(k+1)$.

Theorem 4.1 tells us that weighted groupoids are the objects that integrate weighted algebroids, but of course it does not tell us that we can actually (globally) integrate weighted Lie algebroids. As a 'corollary' we see that $\mathcal{V B}$-groupoids are the objects that integrate $\mathcal{V} \mathcal{B}$ algebroids.

Example 4.2. The weighted Lie algebroid associated with the weighted pair Lie groupoid $F_{k} \times F_{k} \rightrightarrows F_{k}$ is the tangent bundle $\mathrm{T} F_{k}$.

Example 4.3. The $k$-th order tangent bundle of a Lie groupoid $\mathrm{T}^{k} \mathcal{G}$ naturally comes with the structure of a weighted Lie groupoid over $\mathrm{T}^{k} M$. The associated Lie algebroid is $\mathrm{A}\left(\mathrm{T}^{k} \mathcal{G}\right) \simeq$ $\mathrm{T}^{k} \mathrm{~A}(\mathcal{G})$, see for example [24] for details. From [3] we know that that $\mathrm{T}^{k} \mathrm{~A}(\mathcal{G})$ is a weighted Lie algebroid.

Proposition 4.4. Via the appropriate forgetful functors, the Lie functor restricts to the subcategories of weighted Lie groupoids and weighted Lie algebroids, i.e.,

$$
\text { wGrpd } \stackrel{\text { Lie }}{\longrightarrow} \text { wAlgd. }
$$

Proof. This follows from Theorem 4.1 and the fact that both weighted Lie groupoid and weighted Lie algebroid morphisms intertwine the respective actions of the homogeneity structure.

\subsection{Integration of weighted Lie algebroids}

We will not address the full problem of integration of Lie algebroids here, one should consult Crainic and Fernandes [7] for details of the obstruction to (global) integrability. For a details of the obstruction to the integration of $\mathcal{V B}$-algebroids see [1]. We remark though, that passing to the world of differentiable stacks and the notion of a Weinstein groupoid all Lie algebroids are integrable [42], though in this work we will strictly stay in the world of Lie groupoids. The question we address is not one of the integrability of weighted Lie algebroids as Lie algebroids, but rather if they integrate to weighted Lie groupoids. That is, does the homogeneity structure on the weighted Lie algebroid integrate to a homogeneity structure on the associated source simply-connected Lie groupoid such that we have the structure of a weighted Lie groupoid?

Theorem 4.5. Let $D_{k+1} \rightarrow B_{k}$ be a weighted Lie algebroid of degree $k+1$ with respect to a homogeneity structure $\widehat{\mathrm{h}}$ and $\Gamma_{k}$ its source simply-connected integration groupoid. Then $\Gamma_{k}$ is a weighted Lie groupoid of degree $k$ with respect to the homogeneity structure $\mathrm{h}$ uniquely determined by (4.1).

Proof. A weighted Lie algebroid is a Lie algebroid together with a homogeneity structure $\widehat{\mathrm{h}}_{t}: D_{k+1} \rightarrow D_{k+1}$ such that for any $t \neq 0$ the homogeneity structure acts as a Lie algebroid automorphism, and for $t=0$ the homogeneity structure is a Lie algebroid morphism to the underlying non-graded Lie algebroid. Thus we can use Lie II to uniquely integrate $\widehat{\mathrm{h}}_{t}$ (for a given $t$ ) to a Lie groupoid morphism

$$
\mathrm{h}_{t}: \Gamma \rightarrow \Gamma
$$

such that $\left(\mathrm{h}_{t}\right)^{\prime}=\widehat{\mathrm{h}}_{t}$. The corresponding action $\mathrm{h}: \mathbb{R} \times \Gamma \rightarrow \Gamma$ is smooth. Indeed, the smooth action $\widehat{\mathrm{h}}$ on the Lie algebroid $D_{k+1}$ induces clearly a smooth action on the Banach manifold of 
$C^{1}$-admissible paths in $D_{k+1}$ whose projection on $\Gamma$ gives h. The uniqueness of the integration of Lie algebroid morphisms together with the functorial properties of differentiating Lie groupoids, namely $\left(\mathrm{h}_{t} \circ \mathrm{h}_{s}\right)^{\prime}=\mathrm{h}_{t}^{\prime} \circ \mathrm{h}_{s}^{\prime}$, implies that $\mathrm{h}: \mathbb{R} \times \Gamma \rightarrow \Gamma$ is a homogeneity structure. Thus $\Gamma \rightrightarrows B$ is a weighted Lie groupoid.

The only remaining question is that of the degree of the homogeneity structure $h$. This in fact follows directly from the Lie functor. The degree of the homogeneity structure is a local question and so we can examine everything in local coordinates. In particular, on $\Gamma \rightrightarrows B$ we can employ local coordinates adapted to the source fibration

$$
\left(x_{u}^{\alpha}, Y_{u}^{I}\right),
$$

for $0 \leq u \leq k^{\prime}$, for some $k^{\prime}$ to be determined. As the homogeneity structure commutes with the source, for simplicity we can assume that the weight of both coordinates run over the same range. Following the construction of the associated Lie algebroid, we equip $\mathrm{T} \Gamma$ with homogeneous coordinates

$$
(\underbrace{x_{u}^{\alpha}}_{(u, 0)}, \underbrace{Y_{u}^{I}}_{(u, 0)}, \underbrace{\dot{x}_{u+1}^{\beta}}_{(u, 1)}, \underbrace{\dot{Y}_{u+1}^{J}}_{(u, 1)}),
$$

where we have applied the tangent lift of the homogeneity structure to define the weight of the coordinates on the tangent bundle. The second component is simply the natural weight induced by the vector bundle structure. Locally we obtain $D_{k+1} \rightarrow B_{k}$ by setting $\dot{x}=0$ and $Y=0$.

Note that as $D_{k+1}$ is a graded bundle of degree $k$ (forget the linear structure) and that $(x, \dot{Y})$ give homogeneous coordinates on the weighted Lie algebroid $D_{k+1} \subset \mathrm{T} \Gamma$. The tangent bundle of a graded bundle of degree $k^{\prime}$ is also a graded bundle of degree $k^{\prime}$ (again forgetting the linear structure). Meaning that the weight of the coordinates $\dot{Y}$ on the algebroid are directly inherited from the coordinates $Y$ on the groupoid. Thus we conclude that $k^{\prime}=k$. Upon setting $\Gamma=\Gamma_{k}$ we have established the theorem.

Remark 4.6. The results of Bursztyn et al. [5] on integration of $\mathcal{V B}$-algebroids rely on some technical issues regarding regular homogeneity structures. In particular it is some work to show that regularity is preserved under integration. By considering the larger category of graded bundles and not just vector bundles we bypass this technicality. The fact that integration of a weighted Lie algebroid produces a weighted Lie groupoid is quite clear; the degree of the integrated homogeneity structure then follows from the graded bundle structure of tangent bundle of the integration Lie groupoid rather than any difficult technical arguments. The philosophy is that graded bundles give us a 'little more room to manoeuvre' as compared with just vector bundles.

Because $\mathrm{A}\left(\mathrm{h}_{0}\left(\Gamma_{k}\right)\right)=\widehat{\mathrm{h}}_{0}\left(D_{k+1}\right)$, it is clear that the integrability of $D_{k+1} \rightarrow B_{k}$ as a Lie algebroid implies that $A \rightarrow M$, where $A=\widehat{\mathrm{h}}_{0}\left(D_{k+1}\right)$ is also integrable as a Lie algebroid. The converse is not true and examples of $\mathcal{V B}$-algebroids with integrable base Lie algebroids for which the total Lie algebroid is not integrable exist, see $[1,5]$.

\section{Weighted Poisson-Lie groupoids and their Lie theory}

Following our general ethos that we can construct weighted versions of our favorite geometric structures by simply including a compatible homogeneity structure, we now turn to the notion of weighted Poisson-Lie groups and weighted Lie bi-algebroids. As the propositions in this section follow by mild adaptation of standard proofs by including weights into considerations, we will not generally present details of the proofs. 


\subsection{Weighted Poisson-Lie groupoids}

Definition 5.1. A weighted Poisson-Lie groupoid $\left(\Gamma_{k}, \Lambda\right)$ of degree $k$ is a Poisson-Lie groupoid equipped with a multiplicative homogeneity structure $\underline{\mathrm{h}}: \mathbb{R} \times \Gamma_{k} \rightarrow \Gamma_{k}$ of degree $k$, such that the Poisson structure is of weight $-k$.

Recall that a Poisson-Lie groupoid is a Lie groupoid equipped with a multiplicative Poisson structure; this means that

$$
\operatorname{graph}(\text { mult })=\{(g, h, g \circ h) \mid \underline{s}(g)=\underline{t}(h)\}
$$

is coisotropic inside $\left(\Gamma_{k} \times \Gamma_{k}, \times \Gamma_{k}, \Lambda \oplus \Lambda \oplus-\Lambda\right)$. The associated Poisson bracket on $C^{\infty}\left(\Gamma_{k}\right)$ is of weight $-k$. Weighted Poisson-Lie groupoids should be thought of as the natural generalisation on $\mathcal{P} \mathcal{V B}$-groupoids as first defined by Mackenzie [27]. A $\mathcal{P} \mathcal{V B}$-groupoid is a $\mathcal{V} \mathcal{B}$-groupoid equipped with a compatible Poisson structure.

An alternative, yet equivalent and illuminating way to view weighted Poisson-Lie groupoids is as follows. Let $(G, \Lambda)$ be a Lie groupoid equipped with a Poisson structure, we will temporarily forget the graded structure and place no conditions on the Poisson structure. Associated with the Poisson structure is the fibre-wise map

$$
\Lambda^{\#}: \mathrm{T}^{*} G \rightarrow \mathrm{T} G .
$$

This fibre-wise map is of course completely independent of the groupoid structure on $G$. Note, however, that if $G$ is a Lie groupoid, then both $\mathrm{T}^{*} G \rightrightarrows \mathrm{A}^{*}(G)$ and $\mathrm{T} G \rightrightarrows \mathrm{T} M$ are naturally Lie groupoids [28]. Then one can then define a Poisson-Lie groupoid as a Lie groupoid equipped with a Poisson structure such that the induced map $\Lambda^{\#}$ is a morphism of Lie groupoids.

The only extra requirement for the case of a weighted Poisson-Lie groupoid is that $\Lambda^{\#}: \mathrm{T}^{*} \Gamma_{k}$ $\rightarrow \mathrm{T} \Gamma_{k}$ must be a morphisms of double graded bundles, and this forces the Poisson structure to be of weight $-k$. Furthermore note that $\mathrm{T}^{*} \Gamma_{k}$ and $\mathrm{T} \Gamma_{k}$ are both $\mathcal{G L}$-bundles, cf. Section 2 .

Example 5.2. Rather trivially, any weighted Lie groupoid of degree $k$ is weighted Poisson-Lie groupoid of degree $k$ when equipped with the zero Poisson structure.

Example 5.3. Any Poisson-Lie groupoid can be considered as a weighted Poisson-Lie groupoid of degree 0 by equipping it with the trivial homogeneity structure. Lie groups equipped with multiplicative Poisson structures are specific examples.

Example 5.4. Consider a graded bundle of degree $k$ equipped with a Poisson structure of weight $-k,\left(F_{k}, \Lambda\right)$. The pair groupoid $F_{k} \times F_{k} \rightrightarrows F_{k}$ is a weighted Poisson-Lie groupoid of degree $k$ if we equip $F_{k} \times F_{k}$ with the 'difference' Poisson structure.

Example 5.5. Consider a Poisson-Lie groupoid $(\mathcal{G} \rightrightarrows M, \pi)$, which we can consider as a trivial weighted Poisson-Lie groupoid. Recall that $\mathrm{T}^{k} \mathcal{G} \rightrightarrows \mathrm{T}^{k} M$ is a weighted Lie groupoid of degree $k$. Furthermore, in [24] it was shown that the higher tangent lift of a multiplicative Poisson structure is a multiplicative Poisson structure itself. By inspection we see that Poisson structure lifted to the $k$-th order tangent bundle $\Lambda:=\mathrm{d}_{\mathrm{T}}^{k} \pi$ is of weight $-k$, with respect to the natural homogeneity structure on the higher tangent bundle. Thus $\left(\mathrm{T}^{k} \mathcal{G} \rightrightarrows \mathrm{T}^{k} M, \mathrm{~d}_{\mathrm{T}}^{k} \pi\right)$ is a weighted Poisson-Lie groupoid of degree $k$. This example we consider as the archetypal weighted Poisson-Lie groupoid.

\subsection{Weighted Lie bi-algebroids}

Follow Roytenberg [38], Voronov [44] and Kosmann-Schwarzbach [22] we are lead to the following definition. 
Definition 5.6. A pair of weighted Lie algebroids $\left(D_{k}, D_{k}^{*}\right)$ both of degree $k$ is a weighted Lie bi-algebroid if and only if their exists odd Hamiltonians $\mathcal{Q}_{D_{k}}$ and $S_{D_{k}^{*}}$ on T* $\left(\Pi D_{k}\right)$ of weight $(k-1,2,1)$ and $(k-1,1,2)$, respectively, that Poisson commute.

Let us employ homogeneous local coordinates

$$
(\underbrace{x_{u}^{\alpha}}_{(u, 0,0)}, \underbrace{\theta_{u+1}^{I}}_{(u, 1,0)}, \underbrace{p_{\beta}^{u+2}}_{(u, 1,1)}, \underbrace{\chi_{J}^{u+1}}_{(u, 0,1)}),
$$

on $\mathrm{T}^{*}\left(\Pi D_{k}\right)$. In these homogeneous coordinates we have

$$
\begin{aligned}
& \mathcal{Q}=\theta_{u-u^{\prime}+1}^{I} Q_{I}^{\alpha}\left[u^{\prime}\right](x) p_{\alpha}^{k+1-u}+\frac{1}{2} \theta_{u^{\prime \prime}-u^{\prime}+1}^{I} \theta_{u-u^{\prime \prime}+1}^{J} Q_{J I}^{K}\left[u^{\prime}\right](x) \chi_{K}^{k-u}, \\
& S=\chi_{I}^{u-u^{\prime}+1} Q^{I \alpha}\left[u^{\prime}\right](x) p_{\alpha}^{k+1-u}+\frac{1}{2} \chi_{I}^{u^{\prime \prime}-u^{\prime}+1} \chi_{J}^{u-u^{\prime \prime}+1} Q_{K}^{J I}\left[u^{\prime}\right](x) \theta_{k-u}^{K}
\end{aligned}
$$

Since we have a Schouten structure here of tri-weight $(k-1,1,2)$, we have a graded fibre-wise map

$$
S^{\#}: \mathrm{T}^{*}\left(\Pi D_{k}\right) \longrightarrow \Pi \mathrm{T}\left(\Pi D_{k}\right)
$$

given in local coordinates by

$$
\left(S^{\#}\right)^{*} \mathrm{~d} x_{u+1}^{\alpha}=\frac{\partial S}{\partial p_{\alpha}^{k+1-u}}, \quad\left(S^{\#}\right)^{*} \mathrm{~d} \theta_{u+2}^{I}=\frac{\partial S}{\partial \chi_{\alpha}^{k-u}},
$$

where we have employed homogeneous local coordinates

$$
(\underbrace{x_{u}^{\alpha}}_{(u, 0,0)}, \underbrace{\theta_{u+1}^{I}}_{(u, 1,0)}, \underbrace{\mathrm{d} x_{u+1}^{\beta}}_{(u, 0,1)}, \underbrace{\mathrm{d} \theta_{u+2}^{J}}_{(u, 1,1)}),
$$

on $\Pi \mathrm{T}\left(\Pi D_{k}\right)$.

Proposition 5.7. Let $\left(D_{k}, D_{k}^{*}\right)$ be a weighted Lie bi-algebroid of degree $k$. The fibre-wise map $S^{\#}$ is a graded morphisms of $Q$-manifolds

$$
\left(\mathrm{T}^{*}\left(\Pi D_{k}\right), Q_{0}:=\{\mathcal{Q}, \bullet\}\right) \longrightarrow\left(\Pi \mathrm{T}\left(\Pi D_{k}\right), \mathcal{L}_{Q}=\left[\mathrm{d}, i_{Q}\right]\right) .
$$

Proof. It follows via direct computation and so we omit details.

Remark 5.8. The above proposition holds for general $Q S$-manifolds and the additional gradings play no critical role. In fact, we do not need a Schouten structure, but just an odd quadratic Hamiltonian such that $\mathcal{L}_{Q} S:=\{\mathcal{Q}, S\}=0$.

Example 5.9. If $\left(\mathrm{A}, \mathrm{A}^{*}\right)$ is a Lie bi-algebroid, then $\left(\mathrm{T}^{k} \mathrm{~A}, \mathrm{~T}^{k} \mathrm{~A}^{*}\right)$ is a Lie bi-algebroid of degree $k+1$. See for details [24], although the authors do not make mention of the graded structure.

Example 5.10. Consider a weighted Lie algebroid $D_{k}$ of degree $k$, together with an even function $\mathcal{P} \in C^{\infty}\left(\Pi D_{k}^{*}\right)$ of bi-weight $(k-1,2)$ such that $\llbracket \mathcal{P}, \mathcal{P} \rrbracket=0$, where the bracket is the Schouten bracket encoding the Lie algebroid structure. Such functions we consider as Poisson structures on weighted Lie algebroids. Then, following well-known classical results, $\left(D_{k}, D_{k}^{*}\right)$ is a weighted Lie bi-algebroid of degree $k$. This example is the weighted version of a triangular Lie bi-algebroid. As a canonical example, $\left(\mathrm{T}\left(\mathrm{T}^{k-1} M\right), \mathrm{T}^{*}\left(\mathrm{~T}^{k-1} M\right)\right)$ is a weighted Lie bi-algebroid of degree $k$. The homological vector field is supplied by the de Rham differential on $\Pi \mathrm{T}\left(\mathrm{T}^{k-1} M\right)$ and the Schouten structure is that associated with the canonical Schouten bracket on $\Pi \mathrm{T}^{*}\left(\mathrm{~T}^{k-1} M\right)$. 


\subsection{The Lie theory of weighted Poisson-Lie groupoids and weighted Lie bi-algebroids}

It is well known that the infinitesimal object associated with a Poisson-Lie groupoid is a Lie bi-algebroid. Let us quickly sketch the association of a Lie bi-algebroid with a Poisson-Lie groupoid. The construction of the underlying Lie algebroid is as standard, we only need to show how the appropriate Schouten structure is generated and that it carries the correct weight. With this in mind, let us outline the classical construction.

It can be shown that there are two natural isomorphisms

$$
\Pi \vartheta: \Pi \mathrm{A}\left(\mathrm{T}^{*} G\right) \longrightarrow \mathrm{T}^{*}(\Pi \mathrm{A}(G)), \quad \Pi j: \Pi \mathrm{T}(\Pi \mathrm{A}(G)) \longrightarrow \Pi \mathrm{A}(\mathrm{T} G)
$$

which follow from application of the parity reversion functor to the standard non-super isomorphisms (see $[28,30]$ and [24], where the higher tangent versions are also discussed). Let $(G, \Lambda)$ be a Poisson-Lie groupoid, then as we have $\Lambda^{\#}: \mathrm{T}^{*} G \rightarrow \mathrm{T} G$, we also have the 'superised' version:

$$
\Pi\left(\Lambda^{\#}\right)^{\prime}: \quad \Pi \mathrm{A}\left(\mathrm{T}^{*} G\right) \rightarrow \Pi \mathrm{A}(\mathrm{T} G) .
$$

Furthermore, the above gives rise to a Schouten structure on A viz

$$
\Pi\left(\Lambda^{\#}\right)^{\prime}:=\Pi j \circ S^{\#} \circ \Pi \vartheta
$$

where $S^{\#}: \mathrm{T}^{*}(\Pi \mathrm{A}(G)) \longrightarrow \Pi \mathrm{T}(\Pi \mathrm{A}(G))$. One now only has to check the weight when dealing with the weighted versions.

In the other direction, it is also well known an integration procedure for passing from Lie bialgebroids to Poisson-Lie groupoids. Using Lie's second theorem one can show that if $\left(A, A^{*}\right)$ is a Lie bi-algebroid and $G \rightrightarrows M$ is the source simply-connected Lie groupoid integrating $A \rightarrow M$, then there exists a unique Poisson structure on $G$ such that $(G, \Lambda)$ is a Poisson-Lie groupoid integrating $\left(A, A^{*}\right)$. Again, it is straightforward to check the weights when dealing with the weighted versions. We are lead to the following proposition.

Proposition 5.11. There is a canonical correspondence between weighted Poisson-Lie groupoids of degree $k$ and (integrable) weighted Lie bi-algebroids of degree $k+1$.

\subsection{The Courant algebroid associated with weighted Lie bi-algebroids}

Following our general ethos, a weighted Courant algebroid is a Courant algebroid (see [6, 25]) with an additional compatible homogeneity structure.

Definition 5.12. A weighted Courant algebroid of degree $k$ consists of the following data:

1. A double graded supermanifold $(\mathcal{M}, \mathrm{h}, \mathrm{l})$ of bi-degree $(k-1,2)$ such that $(\mathcal{M}, \mathrm{l})$ is an $N$-manifold (the Grassmann parity of homogeneous coordinates is determined by $\mathrm{l}$ ).

2. An even symplectic form $\omega$ on $\mathcal{M}$ of bi-degree $(k-1,2)$.

3. An odd function $\Theta \in C^{\infty}(\mathcal{M})$ of bi-degree $(k-1,3)$, such that $\{\Theta, \Theta\}_{\omega}=0$, where the bracket is the Poisson bracket induced by $\omega$.

The corresponding derived bracket

$$
\llbracket \sigma^{1}, \sigma^{2} \rrbracket_{\Theta}:=\left\{\left\{\sigma^{1}, \Theta\right\}_{\omega}, \sigma^{2}\right\}_{\omega}
$$

is closed on functions of bi-degree $(k-1,1)$ (so functions of total degree $k$ ) and defines the higher analog of the Courant-Dorfman bracket. 
Our motivating example is associated with an arbitrary weighted Lie bi-algebroid $\left(D_{k}, D_{k}^{*}\right)$. Let us 'collapse' the tri-weight on $\mathrm{T}^{*}\left(\Pi D_{k}\right)$ to a bi-weight by taking the sum of the last two weight vector fields. Then, the Hamiltonian vector field

$$
Q_{\lambda}:=\{\mathcal{Q}+\lambda S, \bullet\} \in \operatorname{Vect}\left(\mathrm{T}^{*}\left(\Pi D_{k}\right)\right)
$$

is of bi-weight $(0,1)$. Here we take $\lambda \in \mathbb{R}$ to be some free parameter (carrying no weight) and so we have a pencil of such vector fields. Clearly $Q_{\lambda}$ is a homological vector field. Moreover, as $Q_{\lambda}$ is a Hamiltonian vector field its Lie derivative annihilates the canonical symplectic structure on $\mathrm{T}^{*}\left(\Pi D_{k}\right)$. Note that the canonical symplectic form has natural bi-weight $(k-1,2)$. Thus, following Roytenberg [38], we have objects that deserve to be called weighted Courant algebroids of degree $k$.

To uncover the natural pairing, bracket and anchor structure, first note that $\Pi D_{k} \times{ }_{B_{k-1}} \Pi D_{k}^{*}$ is a quotient supermanifold of $\mathrm{T}^{*} \Pi D_{k}$ defined (locally) by projecting out the coordinates $p_{\alpha}^{u+1}$. Thus we will naturally identify functions on $\mathrm{T}^{*}\left(\Pi D_{k}\right)$ of bi-weight $(r-1,1)$ as homogeneous sections of the vector bundle $D_{k} \oplus D_{k}^{*}=D_{k} \oplus_{B_{k-1}} D_{k}^{*}$ of degree $r$, up to a shift in the Grassmann parity. In the homogeneous coordinates introduced above, any homogeneous section of degree $r$ is of the form

$$
\sigma_{r}=s_{r-u-1}^{I}(x) \chi_{I}^{u+1}+\theta_{u+1}^{I} s_{I}^{r-u-1}(x) .
$$

The natural pairing between sections, that is a fibre-wise pseudo-Riemannian structure on $D_{k} \oplus D_{k}^{*}$, comes from the Poisson bracket on $\mathrm{T}^{*}\left(\Pi D_{k}\right)$,

$$
\left\langle\sigma^{1}, \sigma^{2}\right\rangle=\left\{\sigma^{1}, \sigma^{2}\right\} \in C^{\infty}\left(B_{k-1}\right)
$$

and thus the pairing carries bi-weight $(-k+1,-2)$, or we can think of the total weight as $-k-1$. The fact that we have identified homogeneous sections with particular odd functions on $\mathrm{T}^{*}\left(\Pi D_{k}\right)$ does not effect the identification of the metric structure and the Poisson structure; everything is linear and so the parity reversion does not add any real complications here. Let us simplify notation slightly and define $\Theta_{\lambda}:=\mathcal{Q}+\lambda S$. Then the derived bracket, given by

$$
\llbracket \sigma^{1}, \sigma^{2} \rrbracket_{\lambda}:=\left\{\left\{\sigma^{1}, \Theta_{\lambda}\right\}, \sigma^{2}\right\},
$$

carries bi-weight $(-k+1,-1)$ (so the total weight $-k$ ), similarly to the case of a weighted Lie algebroid. It is closed on functions of bi-degree $(k-1,1)$, so sections of the vector bundle $D_{k} \oplus D_{k}^{*}$ of degree $k$. The latter is the 'higher' analog of the Courant-Dorfman bracket, which is not a Lie bracket, but rather a Loday (Leibniz) bracket. The anchor is then defined as

$$
\rho_{\lambda}: \operatorname{Sec}\left(D_{k} \oplus D_{k}^{*}\right) \rightarrow \operatorname{Vect}\left(B_{k-1}\right), \quad \rho_{\lambda}(\sigma)[f]:=\left\{\left\{\sigma, \Theta_{\lambda}\right\}, f\right\},
$$

for all $f \in C^{\infty}\left(B_{k-1}\right)$.

Theorem 5.13. Via the above construction, if $\left(D_{k}, D_{k}^{*}\right)$ is a weighted Lie bi-algebroid of degree $k$, then $\mathrm{T}^{*}\left(\Pi D_{k}\right)$ naturally has the structure of a weighted Courant algebroid of degree $k$ ( setting $\lambda=1$ for simplicity).

Example 5.14. The $k=1$ case is just that of the Courant structure associated with a standard Lie bi-algebroid. The case of $k=2$ gives an example of a $\mathcal{V B}$-Courant algebroid.

Example 5.15. As any weighted Lie algebroid $D_{k}$ can be considered as a weighted Lie bialgebroid with the trivial structure on the dual bundle, $\mathrm{T}^{*}\left(\Pi D_{k}\right)$ can be considered as a weighted Courant algebroid with the obvious homological vector field. 
Example 5.16. If we take $D_{k}=\mathrm{T}\left(\mathrm{T}^{k-1} M\right)$ then we have the natural structure of a weighted Courant algebroid given in local coordinates as

$$
Q=\left\{\theta_{u+1}^{\alpha} p_{\alpha}^{k+1-u}, \bullet\right\}=\theta_{u+1}^{\alpha} \frac{\partial}{\partial x_{u}^{\alpha}}+p_{\alpha}^{u+1} \frac{\partial}{\partial \chi_{\alpha}^{u+1}} \in \operatorname{Vect}\left(\mathrm{T}^{*}\left(\Pi \mathrm{T}\left(\mathrm{T}^{k-1} M\right)\right)\right) .
$$

This example should be considered as an natural generalisation of the canonical Courant algebroid on the generalised tangent bundle $\mathrm{T} M \oplus \mathrm{T}^{*} M$, which is a substructure of $\mathrm{T}\left(\mathrm{T}^{*} M\right)$.

\subsection{Remarks on contact and Jacobi groupoids}

The notion of a weighted symplectic groupoid is clear: it is just a weighted Poisson groupoid with an invertible Poisson, thus symplectic, structure. By replacing the homogeneity structure, i.e., an action of the monoid $\mathbb{R}$ of multiplicative reals, with a smooth action of its subgroup $\mathbb{R}^{\times}=\mathbb{R} \backslash\{0\}$, one obtains a principal $\mathbb{R}^{\times}$-bundle in the category of symplectic (in general Poisson) groupoids. We get in this way the 'proper', in our belief, definition of a contact (resp. Jacobi) groupoid.

This belief is based on the general and well-known credo, presented, e.g., in [12], that the geometry of contact (more generally, Jacobi) structures on a manifold $M$ is nothing but the geometry of 'homogeneous symplectic' (resp. 'homogeneous Poisson') structures on an $\mathbb{R}^{\times}$-principal bundle $P \rightarrow M$. The word 'homogeneous' refers to the fact that the symplectic form (resp., Poisson tensor) is homogeneous with respect to the $\mathbb{R}^{\times}$-action. Of course, it is pretty well known that any contact (resp. Jacobi) structure admits a symplectization (resp. poissonization) and these facts are frequently used in proving theorems on contact and Jacobi structures (see, e.g., [35]). However, in [12] the symplectization/poissonization was taken seriously as a genuine definition of a contact (Jacobi) structure. The general credo then says that any object related to a contact (Jacobi) structure ought to be considered as the corresponding object in the symplectic (Poisson) category, equipped additionally with an $\mathbb{R}^{\times}$-principal bundle structure, compatible with other structures defining the object. The only problem is what the compatibility means in various cases.

For Lie groupoids (and also Lie algebroids) is natural to expect that the condition of an $\mathbb{R}^{\times}$action to be compatible with the groupoid (algebroid) structure is the same as for the action of multiplicative $\mathbb{R}$, i.e., it consists of groupoid (algebroid) isomorphisms. This is the reason why we comment on contact and Jacobi groupoids here. We present the detailed study of contact/Jacobi groupoids in a separate paper [4].

At this point, note only that our definition of a contact groupoid turns out to be equivalent to the definition of Dazord [10]. The first and frequently used definition, presented in [18], is less general and involves an arbitrary multiplicative function, that is due to the fact that in this approach contact structures are forced to be trivial, i.e., to admit a global contact 1-form. Note that very similar idea of using 'homogeneous symplectic' manifolds in the context of contact groupoids appears already in [9] in a slightly less general framework of $\mathbb{R}_{+}$-actions.

To finish, we want to stress that our use of the group $\mathbb{R}^{\times}$rather than $\mathbb{R}_{+}$is motivated by the need of including non-trivial line bundles into the picture; there is a nice one-to-one correspondence between principal $\mathbb{R}^{\times}$-bundles and line bundles. In this understanding, the contact structure does not need to admit a global contact form and the Jacobi bracket is defined on sections of a line bundle rather than on functions, so it is actually a local Lie algebra in the sense of Kirillov [19] or a Jacobi bundle in the sense of Marle [31]. This, in turn, comes from the observation (cf. [12, Remark 2.4]) that the Jacobi bracket is par excellence a Lie bracket related to a module structure, even if the regular module action of an associative commutative algebra on itself looks formally as the multiplication in the algebra. Consequently, allowing nontrivial modules (line bundles) is necessary for the full and correct picture. 


\section{Acknowledgements}

The authors are indebted to the anonymous referees whose comments have served to improve the content and presentation of this paper. The research of K. Grabowska and J. Grabowski was funded by the Polish National Science Centre grant under the contract number DEC2012/06/A/ST1/00256. A.J. Bruce graciously acknowledges the financial support of the Warsaw Centre for Mathematics and Computer Science in the form of a postdoctoral fellowship.

\section{References}

[1] Brahic O., Cabrera A., Ortiz C., Obstructions to the integrability of $\mathcal{V B}$-algebroids, arXiv:1403.1990.

[2] Bruce A.J., Grabowska K., Grabowski J., Higher order mechanics on graded bundles, J. Phys. A: Math. Theor. 48 (2015), 205203, 32 pages, arXiv:1412.2719.

[3] Bruce A.J., Grabowska K., Grabowski J., Linear duals of graded bundles and higher analogues of (Lie) algebroids, arXiv:1409.0439.

[4] Bruce A.J., Grabowska K., Grabowski J., Remarks on contact and Jacobi geometry, arXiv:1507.05405.

[5] Bursztyn H., Cabrera A., del Hoyo M., Vector bundles over Lie groupoids and algebroids, arXiv:1410.5135.

[6] Courant T.J., Dirac manifolds, Trans. Amer. Math. Soc. 319 (1990), 631-661.

[7] Crainic M., Fernandes R.L., Integrability of Lie brackets, Ann. of Math. 157 (2003), 575-620, math.DG/0105033.

[8] Crainic M., Fernandes R.L., Lectures on integrability of Lie brackets, in Lectures on Poisson geometry, Geom. Topol. Monogr., Vol. 17, Geom. Topol. Publ., Coventry, 2011, 1-107, math.DG/0611259.

[9] Crainic M., Zhu C., Integrability of Jacobi and Poisson structures, Ann. Inst. Fourier (Grenoble) 57 (2007), 1181-1216, math.DG/0403268.

[10] Dazord P., Intégration d'algèbres de Lie locales et groupoïdes de contact, C. R. Acad. Sci. Paris Sér. I Math. 320 (1995), 959-964.

[11] Grabowski J., Quasi-derivations and QD-algebroids, Rep. Math. Phys. 52 (2003), 445-451, math.DG/0301234.

[12] Grabowski J., Graded contact manifolds and contact Courant algebroids, J. Geom. Phys. 68 (2013), 27-58, arXiv:1112.0759.

[13] Grabowski J., Grabowska K., Urbański P., Geometry of Lagrangian and Hamiltonian formalisms in the dynamics of strings, J. Geom. Mech. 6 (2014), 503-526, arXiv:1401.6970.

[14] Grabowski J., Rotkiewicz M., Higher vector bundles and multi-graded symplectic manifolds, J. Geom. Phys. 59 (2009), 1285-1305, math.DG/0702772.

[15] Grabowski J., Rotkiewicz M., Graded bundles and homogeneity structures, J. Geom. Phys. 62 (2012), 21-36, arXiv:1102.0180.

[16] Gracia-Saz A., Mehta R.A., Lie algebroid structures on double vector bundles and representation theory of Lie algebroids, Adv. Math. 223 (2010), 1236-1275, arXiv:0810.0066.

[17] Gracia-Saz A., Mehta R.A., VB-groupoids and representations of Lie groupoids, arXiv:1007.3658.

[18] Kerbrat Y., Souici-Benhammadi Z., Variétés de Jacobi et groupoïdes de contact, C. R. Acad. Sci. Paris Sér. I Math. 317 (1993), 81-86.

[19] Kirillov A.A., Local Lie algebras, Russ. Math. Surv. 31 (1976), no. 4, 55-76.

[20] Kolář I., Michor P.W., Slovák J., Natural operations in differential geometry, Springer-Verlag, Berlin, 1993.

[21] Kontsevich M., Deformation quantization of Poisson manifolds, Lett. Math. Phys. 66 (2003), 157-216, q-alg/9709040.

[22] Kosmann-Schwarzbach Y., Exact Gerstenhaber algebras and Lie bialgebroids, Acta Appl. Math. 41 (1995), 153-165.

[23] Kosmann-Schwarzbach Y., Derived brackets, Lett. Math. Phys. 69 (2004), 61-87, math.DG/0312524.

[24] Kouotchop Wamba P.M., Ntyam A., Tangent lifts of higher order of multiplicative Dirac structures, Arch. Math. (Brno) 49 (2013), 87-104. 
[25] Liu Z.-J., Weinstein A., Xu P., Manin triples for Lie bialgebroids, J. Differential Geom. 45 (1997), 547-574, dg-ga/9508013.

[26] Mackenzie K.C.H., Lie groupoids and Lie algebroids in differential geometry, London Mathematical Society Lecture Note Series, Vol. 124, Cambridge University Press, Cambridge, 1987.

[27] Mackenzie K.C.H., On symplectic double groupoids and the duality of Poisson groupoids, Internat. J. Math. 10 (1999), 435-456, math.DG/9808005.

[28] Mackenzie K.C.H., General theory of Lie groupoids and Lie algebroids, London Mathematical Society Lecture Note Series, Vol. 213, Cambridge University Press, Cambridge, 2005.

[29] Mackenzie K.C.H., Xu P., Lie bialgebroids and Poisson groupoids, Duke Math. J. 73 (1994), 415-452.

[30] Mackenzie K.C.H., Xu P., Integration of Lie bialgebroids, Topology 39 (2000), 445-467, dg-ga/9712012.

[31] Marle C.-M., On Jacobi manifolds and Jacobi bundles, in Symplectic Geometry, Groupoids, and Integrable Systems (Berkeley, CA, 1989), Math. Sci. Res. Inst. Publ., Vol. 20, Springer, New York, 1991, 227-246.

[32] Martínez E., Higher-order variational calculus on Lie algebroids, J. Geom. Mech. 7 (2015), 81-108, arXiv:1501.0652.

[33] Mehta R.A., Supergroupoids, double structures, and equivariant cohomology, Ph.D. Thesis, University of California, Berkeley, 2006, math.DG/0605356.

[34] Mehta R.A., Q-groupoids and their cohomology, Pacific J. Math. 242 (2009), 311-332, math.DG/0611924.

[35] Mehta R.A., Differential graded contact geometry and Jacobi structures, Lett. Math. Phys. 103 (2013), 729-741, arXiv:1111.4705.

[36] Moerdijk I., Mrčun J., On integrability of infinitesimal actions, Amer. J. Math. 124 (2002), 567-593, math.DG/0006042.

[37] Pradines J., Représentation des jets non holonomes par des morphismes vectoriels doubles soudés, C. R. Acad. Sci. Paris Sér. A 278 (1974), 1523-1526.

[38] Roytenberg D., On the structure of graded symplectic supermanifolds and Courant algebroids, in Quantization, Poisson Brackets and Beyond (Manchester, 2001), Contemp. Math., Vol. 315, Amer. Math. Soc., Providence, RI, 2002, 169-185, math.SG/0203110.

[39] Roytenberg D., AKSZ-BV formalism and Courant algebroid-induced topological field theories, Lett. Math. Phys. 79 (2007), 143-159, hep-th/0608150.

[40] Schwarz A., Semiclassical approximation in Batalin-Vilkovisky formalism, Comm. Math. Phys. 158 (1993), 373-396, hep-th/9210115.

[41] Ševera P., Some title containing the words "homotopy" and "symplectic", e.g. this one, Trav. Math. 16 (2005), 121-137, math.SG/0105080.

[42] Tseng H.-H., Zhu C., Integrating Lie algebroids via stacks, Compos. Math. 142 (2006), 251-270, math.DF/0405003.

[43] Vaintrob A.Yu., Lie algebroids and homological vector fields, Russ. Math. Surv. 52 (1997), 428-429.

[44] Voronov Th.Th., Graded manifolds and Drinfeld doubles for Lie bialgebroids, in Quantization, Poisson Brackets and Beyond (Manchester, 2001), Contemp. Math., Vol. 315, Amer. Math. Soc., Providence, RI, 2002, 131-168, math.DG/0105237.

[45] Voronov Th.Th., Q-manifolds and higher analogs of Lie algebroids, in XXIX Workshop on Geometric Methods in Physics, AIP Conf. Proc., Vol. 1307, Amer. Inst. Phys., Melville, NY, 2010, 191-202, arXiv:1010.2503.

[46] Voronov Th.Th., Q-manifolds and Mackenzie theory, Comm. Math. Phys. 315 (2012), 279-310, arXiv:1206.3622. 